\title{
Small-angle neutron scattering on polymer gels: phase behavior, inhomogeneities and deformation mechanisms
}

\author{
Mitsuhiro Shibayama \\ Recent developments in small-angle neutron scattering (SANS) investigations on polymer gels are reviewed by encompassing \\ (i) volume phase transition and microphase separation, (ii) inhomogeneities in polymer gels, (iii) pressure dependence of \\ hydrophobic interaction and (iv) structural characterization of super-tough gels. These developments owe much to the \\ understanding of gel inhomogeneities and advances in the precision analyses of SANS, such as the contrast variation method \\ coupled with singular value decomposition and the accurate evaluation of incoherent scattering intensity. As one of the fruitful \\ outcomes, deformation mechanisms in various types of super-tough gels are elucidated.
}

Polymer Journal (2011) 43, 18-34; doi:10.1038/pj.2010.110; published online 17 November 2010

Keywords: inhomogeneities; polymer gels; $\mathrm{N}$-isopropylacrylamide; small-angle neutron scattering; tetra-PEG gel; tough gel; volume phase transition

\section{INTRODUCTION}

Scattering methods, such as light scattering (LS), X-ray scattering and neutron scattering, have long contributed to the structural characterization of polymeric systems. LS has been used to characterize polymers in solutions since the 1940s. ${ }^{1-3}$ Typical examples are the Guinier plot, Zimm plot and Kratky plot analyses, from which the weight-average molecular weight, the radius of gyration and virial coefficients can be evaluated. ${ }^{4}$ Xenon or mercury light sources were replaced by high-power lasers in the 1970s. ${ }^{3,5}$ This allowed for the quick and accurate determination of the above-mentioned molecular parameters. X-ray scattering techniques have also been greatly advanced after synchrotron sources became available in the 1980s. At present, high-energy brilliant X-ray beams are available for various types of scattering experiments, such as microbeam X-ray scattering, grazing angle X-ray scattering and X-ray reflectivity. ${ }^{6}$

Neutron scattering has several characteristic features, some of which are advantageous to X-ray scattering: ${ }^{7}$ (1) wavelength suitable for nanometer-scale structural analysis (diffraction), (2) sensitivity to elements and their isotopes (nuclear scattering), (3) high penetration power (radiography), (4) capability of labeling without changing the chemical/ physical properties, that is, isotope labeling (H/D substitution), (5) sensitivity to the magnetic structure of the sample (magnetic scattering) and (6) energy exchange between neutron and nucleus in the sample due to the finite (that is, non-zero) mass of the neutron (inelastic scattering). Because of these properties, neutron scattering has been used in the characterization of material structure, magnetic structure and excitation states of condensed matter, and in the dynamics of soft matter. ${ }^{8}$
Among the various types of neutron scattering methodologies, small-angle neutron scattering (SANS) uses only low scattering angles, $\theta$, up to 5 or 10 degrees. Because the typical wavelength of a neutron beam used for SANS is $\lambda_{\mathrm{N}} \approx 0.6-1 \mathrm{~nm}$, the structural characterization of various types of materials and/or the detection of density/concentration fluctuations of media in a spatial range up to $100 \mathrm{~nm}(\approx(4 \pi /$ $\left.\left.\lambda_{\mathrm{N}}\right) \sin (\theta / 2)\right)$ can be conducted by SANS. SANS is a complementary technique to small-angle X-ray scattering ${ }^{6,9}$ and LS., ${ }^{5,10}$ SANS was applied to polymer science in the early 1970 s. ${ }^{11,12}$ Among the various applications of SANS to polymer characterization-namely, polymer solutions, the structure of crystalline morphology, polymer blends and block copolymers-polymer gels are very suitable to being investigated by SANS because of the following reasons. ${ }^{13}$ (1) Scattering contrast can be easily bestowed on the sample simply by producing a gel in a deuterated solvent or by immersing in a deuterated solvent. This also significantly lowers the incoherent scattering arising from $\mathrm{H}$-containing materials. (2) Special sample measurements, such as high-pressure measurements, shear deformation measurements and stretching measurements under temperature and humidity control, can be easily realized with quartz, sapphire and/or metal windows because of the strong penetration power of a neutron beam.

In this review, we introduce the recent advancement of SANS in studying polymer gels by emphasizing the detailed analysis of critical phenomena, microphase separation transition (MiST), inhomogeneities and deformation mechanisms. We first review SANS studies on the volume phase transition (VPT) and MiST of gels. Here, dramatic changes in SANS intensity with temperature, $\mathrm{pH}$ and ionization are 
demonstrated. ${ }^{14,15}$ We then discuss the most important structural aspect of gels, 'inhomogeneities, ${ }^{16}$ which govern the mechanical, optical and other physical properties of gels. Third, we review the applications of SANS to harsh environments, that is, phase behavior of polymeric systems under very high hydrostatic pressures. ${ }^{17-22}$ Here, the roles of hydrophobic interactions in aqueous systems are discussed as a function of pressure. Fourth, the structural characterization of various types of super-tough gels will be shown by focusing on deformation mechanisms. Finally, we conclude this review by addressing future directions of SANS in the structural characterization of polymer gels.

\section{VPT AND MICROPHASE SEPARATION}

VPT is a phase transition observed in polymer gels. ${ }^{23-25}$ The order parameter is the polymer concentration that undergoes a dramatic change at the transition point on a slight change in temperature, solvent volume fraction, $\mathrm{pH}$ or some other intensive property. Poly ( $N$-isopropylacrylamide) (PNIPA) hydrogels are the most extensively studied gels because of their sharp temperature sensitivity around ambient temperature. ${ }^{26-28}$ By adding a small amount of ionizable comonomer, for example, acrylic acid (AAc), a PNIPA gel undergoes a large and discrete VPT. ${ }^{25,29}$ Figure 1 shows a phase diagram of (a) neutral gel and (b) charged gel undergoing VPT, with the corresponding optical micrographs. As shown in Figure 1a, a neutral gel gradually shrinks along the isobar line when it is heated (photographs from left-bottom to right). However, if a gel is heated quickly (temperature jump), it does not have enough time to equilibrate its volume (or its polymer concentration) and undergoes phase separation along an isochore line, as shown in the photograph on the top-left corner. Critical phenomena, that is, the divergence of concentration fluctuations, take place along the isobar line. ${ }^{30}$ SANS investigations of the critical phenomena of PNIPA aqueous solutions and hydrogels were carried out, from which critical exponents close to those of the threedimensional (3D) Ising model were obtained. ${ }^{14}$ Figure $1 \mathrm{~b}$ shows the phase diagram of a weakly charged PNIPA/AAc gel. ${ }^{15}$ In this case, the VPT becomes discrete with a large volume gap similar to that of a gasvapor transition. ${ }^{25}$ In such a weakly charged gel, the coexistence of swollen and shrunken phases can sometimes be observed. The photograph in Figure 1b shows such a 'bi-phase. ${ }^{31,32}$ Figure 2 shows SANS intensity curves of PNIPA/AAc hydrogels observed at various temperatures (left) and an optical photograph showing a hydrogel undergoing collapsed (or swelling) transition on a slight increase (or decrease) in temperature. ${ }^{15}$ Such bi-phases in gels were also observed by Hirotsu. ${ }^{32}$ As shown in the SANS curves, a distinct scattering peak was observed. This indicates large concentration fluctuations in the gel with polymer-rich and -poor domains. Note that, macroscopically, both the swollen and the collapsed phases look homogeneous, but the gel itself is in a highly frustrated state microscopically on the order of a
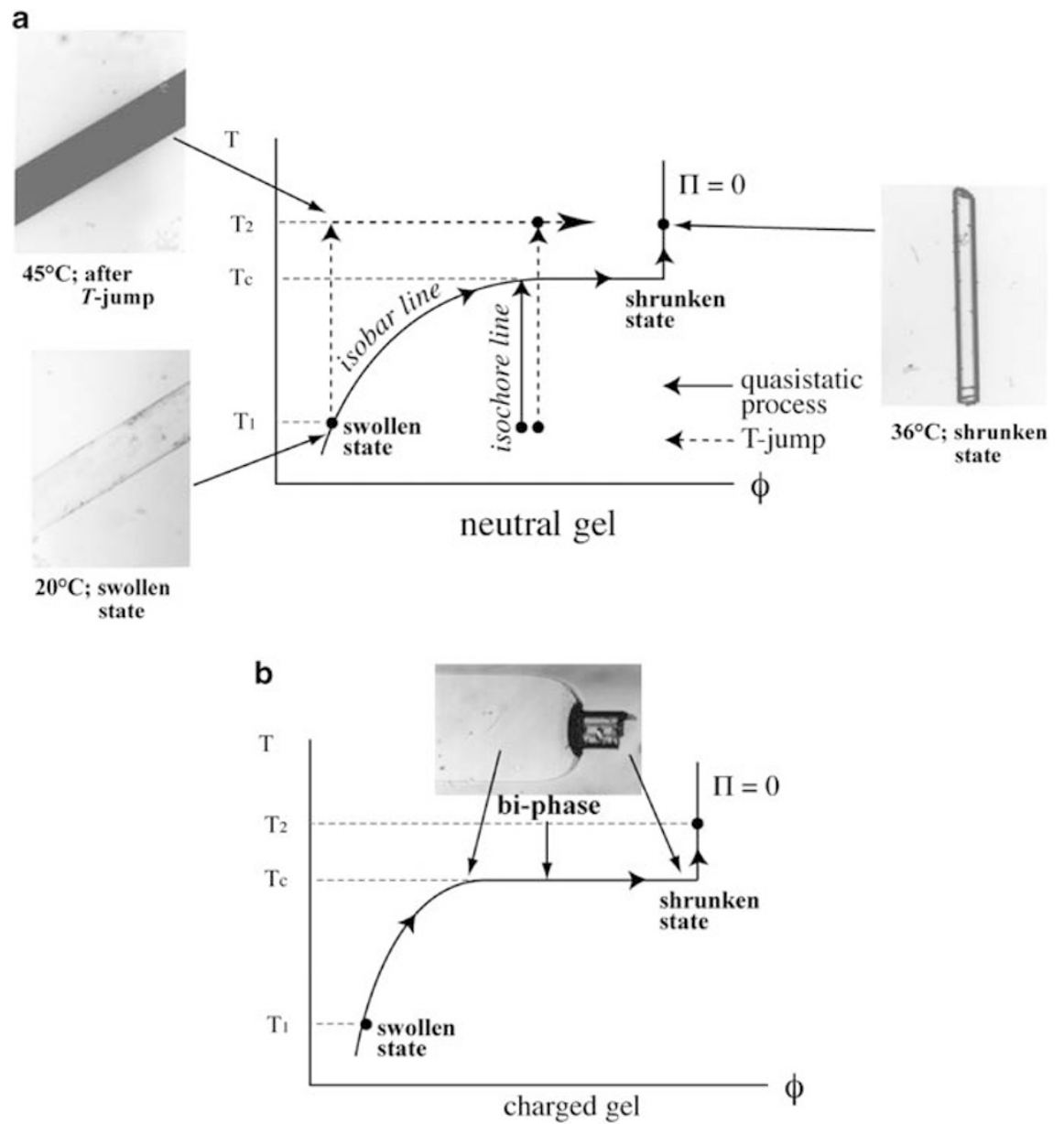

Figure 1 Phase diagram of (a) neutral gel and (b) charged gel undergoing volume phase transition (VPT). Corresponding optical micrographs are shown; swollen gel $\left(20^{\circ} \mathrm{C}\right)$, shrunken gel after $T$-jump $\left(45^{\circ} \mathrm{C}\right)$, shrunken gel in thermal equilibrium $\left(36^{\circ} \mathrm{C}\right)$, and (b) bi-phase gel (bottom). 


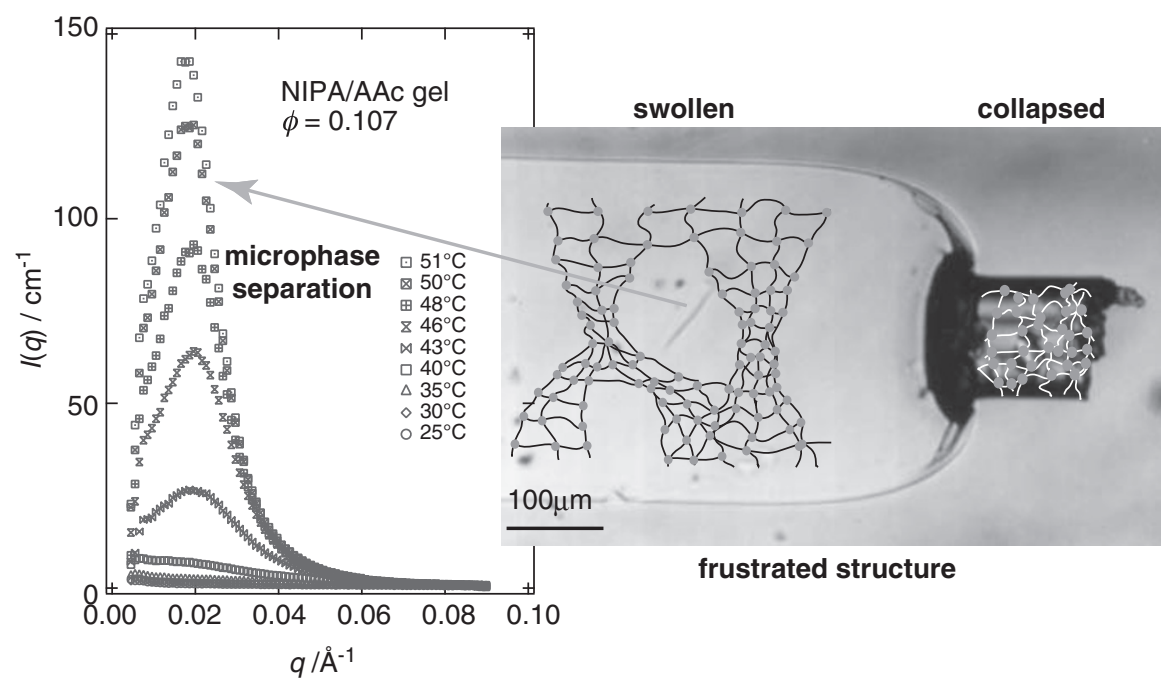

Figure 2 Small-angle neutron scattering (SANS) curves of $N$-isopropylacrylamide/acrylic acid (NIPA/AAc) weakly charged gel observed at various temperatures, together with a photograph showing a swollen and collapsed bi-phase state.

few tens of nanometers. The existence of a scattering peak is an indication of microphase separation in gels. Unlike the microphase separation of block copolymers, ${ }^{33}$ microphase separation in gels does not have various types of morphologies and is simply characterized by the repeat distance of the concentration fluctuations; that is, $2 \pi / q_{\mathrm{m}}$, where $q_{\mathrm{m}}$ is the magnitude of the scattering vector at the scattering maximum. Variations in the repeat distance were investigated as functions of temperature, ${ }^{15} \mathrm{pH},{ }^{34}$ crosslinker concentration ${ }^{35}$ and salt concentration.

\section{INHOMOGENEITIES}

When crosslinkers are introduced to a reaction bath of monomer solutions and/or polymer solutions, an inhomogeneous crosslinking reaction takes place. As a result, inhomogeneities are created in polymer gels. ${ }^{36,37}$ Because polymer chains are tied with chemical bonds, the concentration fluctuations cannot be released and remain as frozen inhomogeneities (that is, permanent concentration fluctuations). Bastide and Leibler ${ }^{38}$ illustrated inhomogeneities in polymer gels with a mesh model, as shown in Figures $3 \mathrm{a}$ and $\mathrm{b}$. Here, blobs of polymer chains and crosslinks are represented by black lines and red dots (colour online). Note that the polymer concentration is above the chain overlap concentration, and crosslinks are randomly introduced in the space. In a reaction bath, concentration fluctuations are suppressed, and the distribution of crosslinks is not detected by scattering. However, when the polymer is swollen, the clusters of first-chemical-neighbor junctions (frozen blobs represented by thick red lines), which do not swell, become 'visible' by scattering. The small clusters, which penetrate large clusters in the reaction bath, are phaseseparated by the swelling of the interstitial medium. As a result, large concentration fluctuations appear by swelling. Scattering is the best way to measure these types of concentration fluctuations, because concentration fluctuations with several modes can be decomposed by Fourier transform. Figure $3 \mathrm{c}$ schematically shows thermal concentration fluctuations, $\rho^{\text {th }}(r)$; (equilibrium) static inhomogeneities introduced by crosslinking reaction, $\rho^{\mathrm{eq}}(r)$; and the sum of these two fluctuations, $\rho(r)$. Panyukov and Rabin ${ }^{39}$ proposed a sophisticated statistical theory of polymer gels by applying a path-integral and replica method. This theory takes into account both thermal and frozen concentration fluctuations. Since then, the quantitative description of inhomogeneities has become possible. According to the Panyukov-Rabin theory, the concentration fluctuations are given by the sum of the thermal and frozen concentration fluctuations:

$$
\rho(r)=\rho^{\mathrm{th}}(r)+\rho^{\mathrm{eq}}(r)
$$

The thermal and frozen structure factors, $G(q)$ and $C(q)$, are given with the Fourier conjugates of $\rho^{\text {th }}(r)$ and $\rho^{\mathrm{eq}}(r)$, respectively, and as

$$
G(q)=\left\langle\rho^{\text {th }}(q) \rho^{\text {th }}(-q)\right\rangle, C(q)=\left\langle\rho^{\mathrm{eq}}(q) \rho^{\mathrm{eq}}(-q)\right\rangle
$$

Here, $\langle X\rangle$ denotes thermal averaging. As a result, the structure factor of gels is given by

$$
S(q) \equiv \overline{\langle\rho(q) \rho(q)\rangle}=G(q)+C(q)
$$

where $\bar{X}$ denotes the ensemble average. Note that the thermal average of $\rho^{\mathrm{eq}}(q)$ is non-zero, that is, $\left\langle\rho^{\mathrm{eq}}(q)\right\rangle \neq 0$, if the system is nonergodic, whereas its ensemble average is always $\overline{\rho^{\mathrm{eq}}(q)}=\overline{\left\langle\rho^{\mathrm{eq}}(q)\right\rangle}=0$ by definition. These functions were obtained for various types of polymer gels, including neutral gels, weakly charged gels and deformed gels. The exact expressions of $G(q), C(q)$ and $S(q)$ are given elsewhere. ${ }^{13,39-42}$

There are various types of inhomogeneities in gels, such as spatial inhomogeneities, topological inhomogeneities and connectivity inhomogeneities. ${ }^{16}$ These inhomogeneities can be characterized by scattering, swelling and/or mechanical measurements and dynamic LS, respectively. SANS is one of the best techniques to study gel inhomogeneities quantitatively because it provides information regarding the spatial concentration fluctuations (in Fourier space) and the concentration differences between solvent-rich and -poor regions.

Figure 4 shows an example of inhomogeneities in SANS and LS profiles. The samples were PNIPA hydrogels prepared with various crosslinker concentrations (bisacrylamide) $C_{\mathrm{BIS}}{ }^{43}$ With increasing $C_{\mathrm{BIS}}$ concentration, the scattering functions exhibit very strong forward scattering at low- $q$. The simplified expressions of $G(q)$ and $C(q)$ are

$$
\begin{aligned}
& G(q) \sim I_{\mathrm{oz}}(q)=\frac{I_{\mathrm{oz}}(0)}{1+\xi^{2} q^{2}} \quad(\text { Ornstein Zernike }) \\
& C(q) \sim I_{\mathrm{SL}}(q)=\frac{I_{\mathrm{SL}}(0)}{\left(1+\Xi^{2} q^{2}\right)^{2}}
\end{aligned}
$$

(squared Lorentz, Debye Bueche)

Here, $\xi$ is the correlation length and $\Xi$ is the characteristic size of the inhomogeneities. 
a

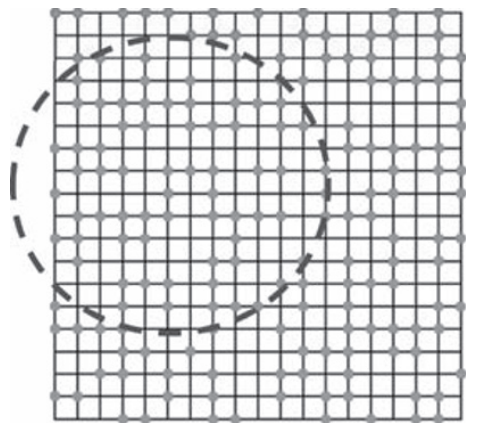

b

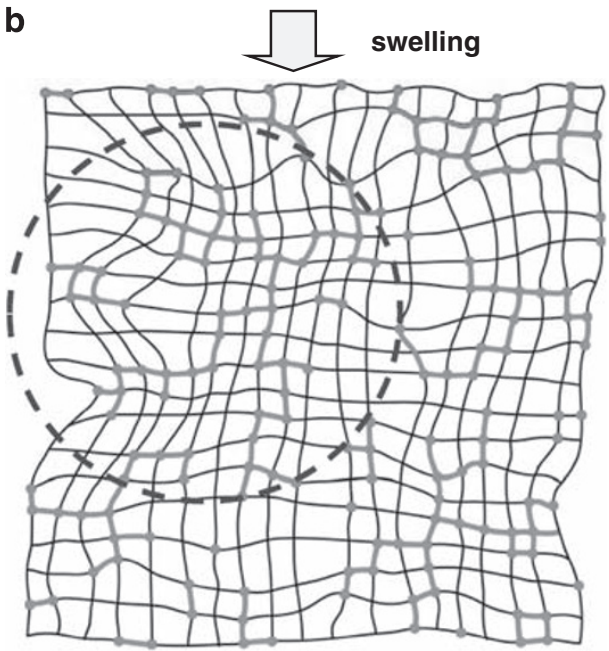

C
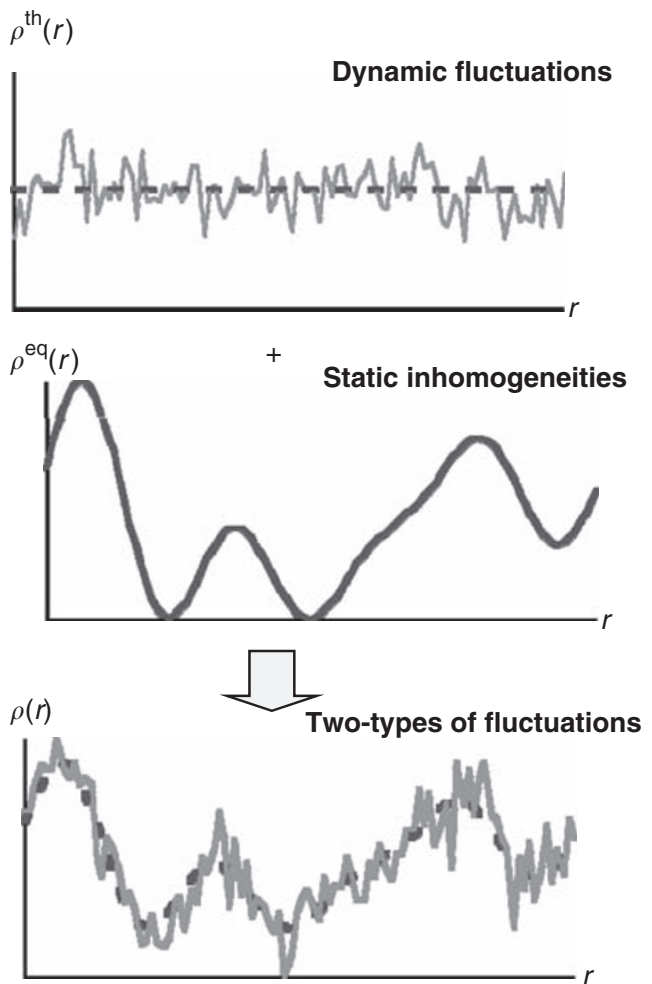

Figure 3 Schematic representations of (a) a two-dimensional reaction bath well above the chain gelation threshold, (b) an overswollen gel by the addition of solvent and (c) dynamic, static and total concentration fluctuations with space coordinate $r$. For the sake of simplicity, the chains, which are random walks on this lattice, are not shown in the figure. Black dots represent the interchain crosslinks placed at random. A full color version of this figure is available at Polymer Journal online.

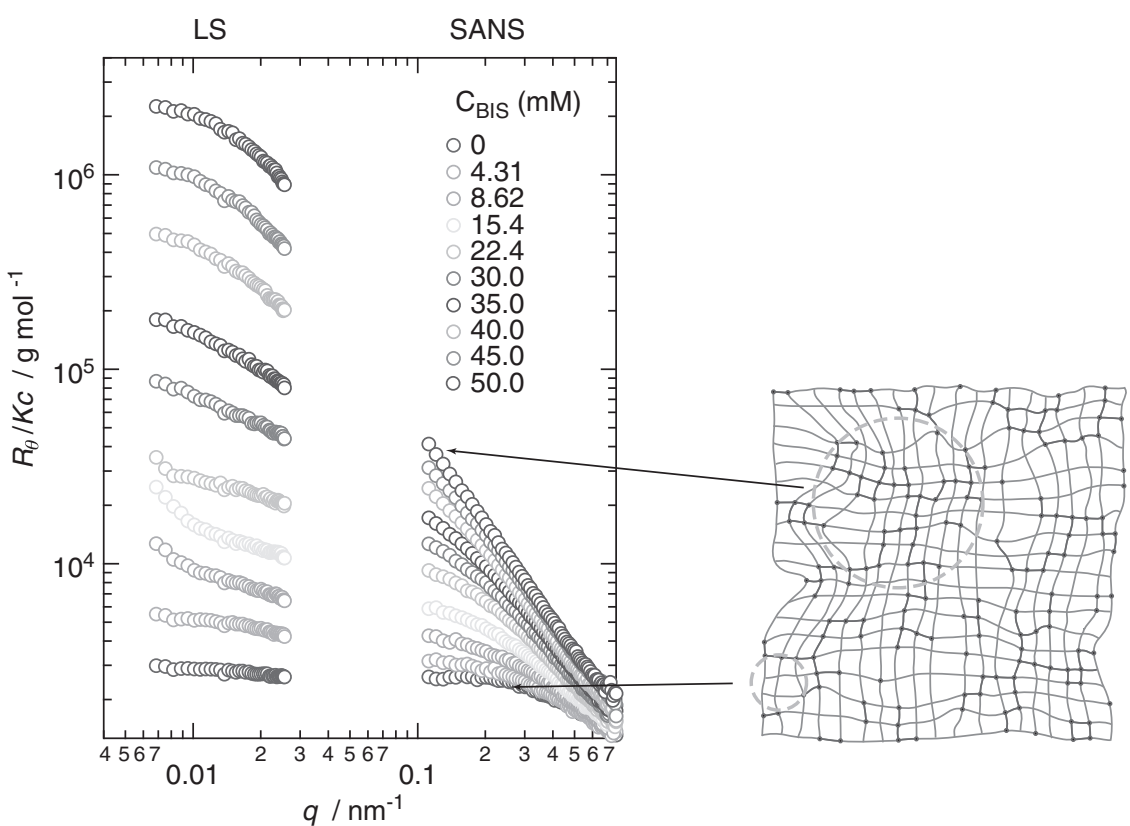

Figure 4 (a) Small-angle neutron scattering (SANS) and light scattering (LS) profiles of PNIPA gels and solution and (b) schematic representation of the gel network (the same as Figure 3b). 


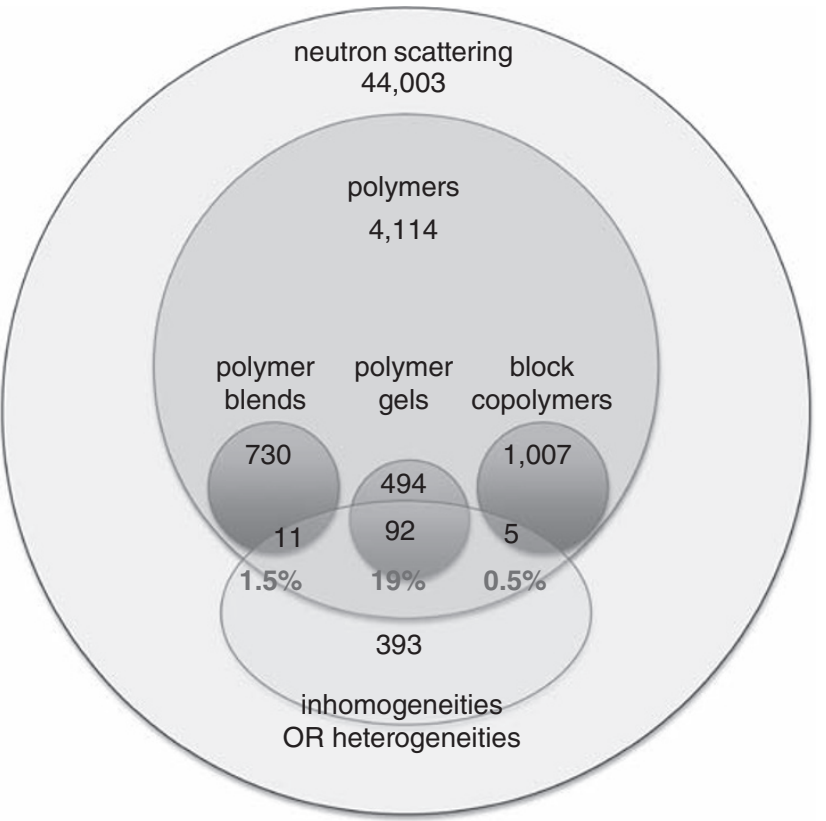

Figure 5 Statistics of small-angle neutron scattering (SANS) papers categorized by various systems: neutron scattering, polymers, polymer blends, polymer gels, block copolymers and inhomogeneities (or heterogeneities) (as of 11 May 2010).

A literature survey of neutron scattering also demonstrates the importance of SANS in characterizing inhomogeneities in polymer gels. Figure 5 shows the statistics of SANS papers categorized by various systems (as of 11 May 2010). The number of papers on neutron scattering is over 44000 , with more than 4000 papers dealing with polymers. These papers are further classified into polymer blends, block copolymers and polymer gels. Thereafter, the number of papers related to inhomogeneities are collected using the AND operation on this term across these systems. The result is given as the numbers and percentages in the figure. Interestingly enough and as expected, scientific papers dealing with SANS, gels and inhomogeneities are the largest among the three systems, that is, polymer blends, block copolymers and polymer gels. This statistic also indicates the importance of inhomogeneities in understanding polymer gels. Until the mid-1990s, studies on inhomogeneities by scattering were carried out using phenomenological theories. ${ }^{37,44}$ However, as mentioned above, the precise analyses of scattering functions for polymer gels became possible because of the Panyukov-Rabin theory ${ }^{39,41}$ and the Onuki theory. ${ }^{45}$ For example, the structures of weakly charged PNIPA/AAc gels were studied in their deformed states ${ }^{46}$ and as functions of $\mathrm{pH} .{ }^{35}$

Deformation mechanisms of polymeric systems have been studied by SANS. ${ }^{47-50}$ Although SANS is a suitable means to study the deformation mechanisms of polymeric systems, chucking a polymer gel onto a stretching device is difficult in most cases because of its fragility. Geissler et al. ${ }^{51}$ used a mold with an elliptical hole and allowed a shrunken gel to swell anisotropically. We created a device to compress a piece of gel from both sides (see Figure 6). A piece of gel was embedded in a hole in a silicon rubber mold. Thereafter, the mold was deformed with screws from both sides, resulting in gel deformation as shown in Figure $6 \mathrm{~b}$. The gel and silicon rubber were kept in a quartz window gap, and deformation in the thickness direction was

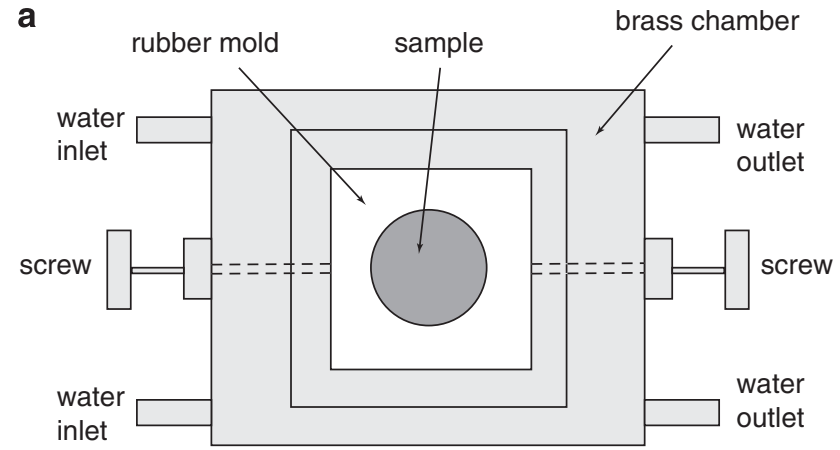

b
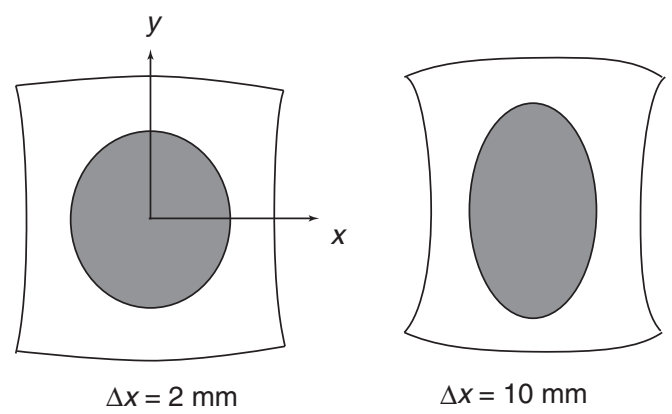

Figure 6 (a) Deformation device of polymer gels for small-angle neutron scattering (SANS) experiment. A piece of gel is embedded in a hole of silicon rubber, which is squeezed from both sides with screws. The silicon rubber and the gel are sandwiched between two pieces of quartz window so as not to deform in the thickness direction. (b) Traces of silicon rubber deformation with the squeezing deformation of 2 and $10 \mathrm{~mm}$.

not allowed. This allowed for gel deformation up to a stretching ratio of $\lambda=1.25$ (that is, $\Delta x=25 \%$ deformation) at various temperatures. Figure 7 shows the 2D-SANS patterns before $(\lambda=1.07)$ and after deformation $(\lambda=1.25)$. At low temperatures, including $26^{\circ} \mathrm{C}$, SANS patterns were rather isotropic, irrespective of the state of deformation. However, anisotropy began to appear in the deformed gel at $42{ }^{\circ} \mathrm{C}$. The scattered intensity patterns at $42{ }^{\circ} \mathrm{C}$ were similar to the so-called butterfly pattern (or, more exactly, the abnormal butterfly pattern). ${ }^{48}$ The scattering intensity contour had a lobe in the stretching direction. At $46^{\circ} \mathrm{C}$, a distinct peak appeared in both undeformed and deformed gels. By deforming the gel, the scattering maximum became elliptical, and $q_{\mathrm{m}}$ moved toward the low- $q$ direction in the stretching direction, whereas the scattered intensity along the equator (that is, along the $x$ direction) seems to have been suppressed. These 2D-SANS patterns were successfully reproduced by the Panykov-Rabin theory. ${ }^{46}$ The theory predicts that deformation results in strong anisotropy in $C(q)$ but not in $G(q)$. This observation may be reasonable if one considers the origins of $C(q)$ and $G(q)$ to be the static inhomogeneity and thermal fluctuations, respectively. A comparison of experimental and theoretical structure factors predicted by the Panyukov-Rabin theory was also made for weakly charged polymer gels as a function of the gel parameters, that is, temperatures at preparation and at observation, polymer concentration and degrees of crosslinking and polymerization. ${ }^{52}$

Another interesting feature of weakly charged gels is the inflection of scattering intensity. Generally, inhomogeneities in gels become larger by increasing the crosslinker concentration. However, in the case of weakly charged gels, an introduction of charges strongly suppresses inhomogeneities. As a result, a crossover in scattering 


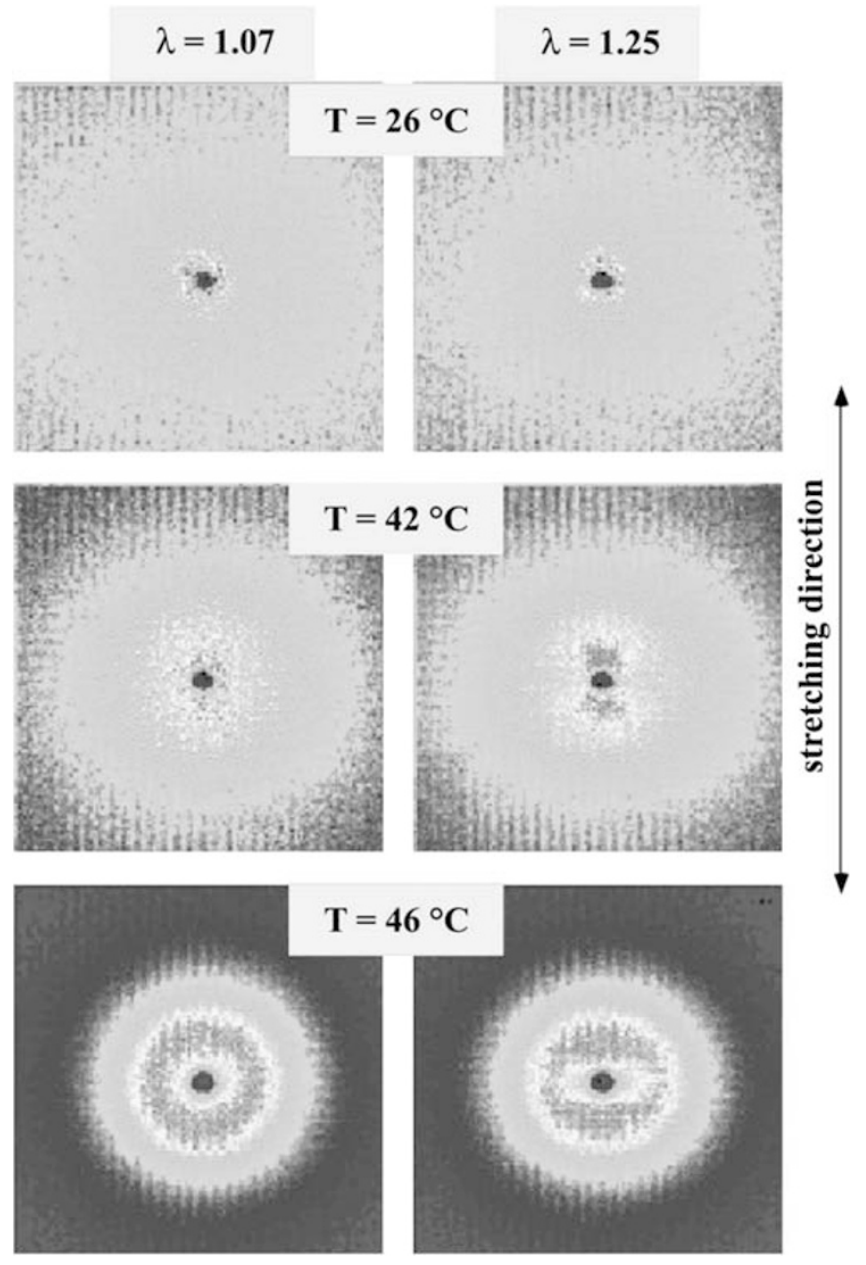

Figure 7 Two-dimensional (2D) small-angle neutron scattering (SANS) patterns of poly( $N$-isopropylacrylamide)/acrylic acid gels before $(\lambda=1.07)$ and after deformation $(\lambda=1.25)$.

intensity with respect to the crosslinking and charges appears at ${ }^{53}$

$$
\chi_{\mathrm{c}}=\frac{1}{2}\left(1+\phi+\frac{f}{\phi}\right)
$$

where $\phi$ and $f$ are the polymer volume fraction and the degree of ionization, respectively. Such inflection was indeed observed in PNIPA/AAc hydrogels. ${ }^{35,54,55}$ This means, in principle, that inhomogeneities can be tuned by adjusting the degrees of crosslinking and ionization.

\section{PHASE BEHAVIORS OF POLYMER GELS AND BLOCK COPOLYMER AQUEOUS SOLUTIONS AT HIGH PRESSURE}

As mentioned in the introduction, SANS allows for structural characterization in harsh environments, such as under high pressure and low temperatures, because of the strong penetration power of neutron beams in comparison with that of X-rays. We performed a series of high-pressure SANS studies on polymer-aqueous systems to elucidate the pressure dependence of the hydrophobic interactions in PNIPA neutral gels, ${ }^{17}$ PNIPA/AAc charged gels, ${ }^{18,19}$ hydrophilic-hydrophobic block copolymer aqueous solutions ${ }^{20-22}$ and globular proteins. ${ }^{56}$ Figure 8 shows a pressure cell for SANS (PCI-400-SANS, Teramex, Kyoto, Japan). The PCI-400-SANS is an inner cell-type pressure cell, which was designed to isolate the sample from the pressurizing medium. The inner cells have a pair of quartz windows. The applied pressure is transmitted through a rubber diaphragm connected to the inner cell. The outer chamber is filled with deuterated water $\left(\mathrm{D}_{2} \mathrm{O}\right)$, and the pressure is controlled by pressurizing $\mathrm{D}_{2} \mathrm{O}$ by a doublecylinder hand pump. ${ }^{57}$ The maximum pressure used was $400 \mathrm{MPa}$.

Figure 9 shows the pressure-temperature phase diagram of poly $(2-$ (2-ethoxy)ethoxyethyl vinyl ether)-block-poly(2-methoxyethyl vinyl ether) (pEOEOVE-pMOVE) in $\mathrm{D}_{2} \mathrm{O}$ and the corresponding SANS patterns. ${ }^{20,22}$ The degrees of polymerization of the individual block chains, that is, pEOEOVE and pMOVE, were 200 and 400, respectively. Both pEOEOVE and pMOVE are soluble in water at low temperatures, but they become insoluble at $\sim 40$ and $65^{\circ} \mathrm{C}$, respectively. Hence, the block copolymer undergoes miscibility transition around these temperatures. A $15.0 \mathrm{wt} \%$ polymer solution was prepared by dissolving pEOEOVE-pMOVE in $\mathrm{D}_{2} \mathrm{O}$. At ambient pressure, the solution underwent a two-step transition at 40 and $65^{\circ} \mathrm{C}$, which are, respectively, assigned to a MiST and macrophase separation transition (MaST), that is, precipitation. Traces of these MiST and MaST were obtained by varying pressure $(P)$ and temperature $(T)$, and the phase diagram shown in Figure 9a was determined by visual observation. The small photographs are the traces of a He-Ne laser beam in a high-pressure cell viewed through a sapphire window. In the one-phase region, the laser beam was difficult to observe. Above the MiST line, weak scattering was observed. On the other hand, in the region above MaST, the sample cell exhibited strong multiple scattering, indicating precipitation of the polymer. Note that both MiST and MaST lines are convex upward functions of pressure with a maximum around $P_{0} \approx 150 \mathrm{MPa}$.

We now discuss the SANS functions in conjunction with the phase diagram. First, we examine the heating process at ambient temperature (Figure 9b). Scattering peaks were clearly visible above $40^{\circ} \mathrm{C}$, indicating MiST. The SANS patterns were reproducible and reversible with respect to temperature. Second, we focus on the case of pressurizing at ambient temperature (Figure 9c). In this case, a gradual increase in scattering intensity occurs in the manner of critical phenomena by pressurizing the gel at room temperature $\left(28^{\circ} \mathrm{C}\right)$. That is, the scattering intensity can be well represented by the Ornstein-Zernike function (see Figure 9c) with the divergence of the correlation length, $\xi$ (see the inset). This pressurization resulted in a MaST with divergence at $350 \mathrm{MPa}$. Third, we discuss the case along the pressure line at $45^{\circ} \mathrm{C}$ (see Figure 9d). Here, a reentrant MiST was observed by increasing pressure. This indicates that states of hydrophobic solvation are different in the low-pressure $\left(P<P_{0}\right)$ and highpressure $\left(P>P_{0}\right)$ regions. Such a reentrant phase diagram was observed not only in water soluble polymers ${ }^{58-60}$ but also in biomacromolecules. ${ }^{61}$ We have discussed the reason for the convexity of the phase diagram in previous work. ${ }^{18,19}$ According to the Clapeyron-Clausius equation, the derivative of pressure with respect to temperature is

$$
\frac{\mathrm{d} P}{\mathrm{~d} T}=\frac{\Delta H_{\mathrm{m}}}{\mathrm{T} \Delta \bar{V}_{\mathrm{m}}}
$$

where $\Delta H_{\mathrm{m}}$ and $\Delta \bar{V}_{\mathrm{m}}$ are the changes in enthalpy and molar volume by mixing, that is, hydrophobic solvation, respectively. The values of $\Delta \bar{V}_{\mathrm{m}}$ at atmospheric pressure were measured by Kato $^{62}$ to be $\Delta \bar{V}_{\mathrm{m}}=-2.4$ and $-2.7 \mathrm{~cm}^{3} \mathrm{~mol}^{-1}$ for PNIPA and ionized PNIPA gels, respectively. Thus, at low pressure, while $\Delta \bar{V}_{\mathrm{m}}$ remains negative, pressurizing pushes the phase separation temperature to higher temperature. The pressure and temperature dependence of the structure factor of PNIPA/AAc gels was reproduced with the Panyukov-Rabin theory and interpreted with a convexity of hydrophobic interaction with respect to pressure. ${ }^{19}$ 


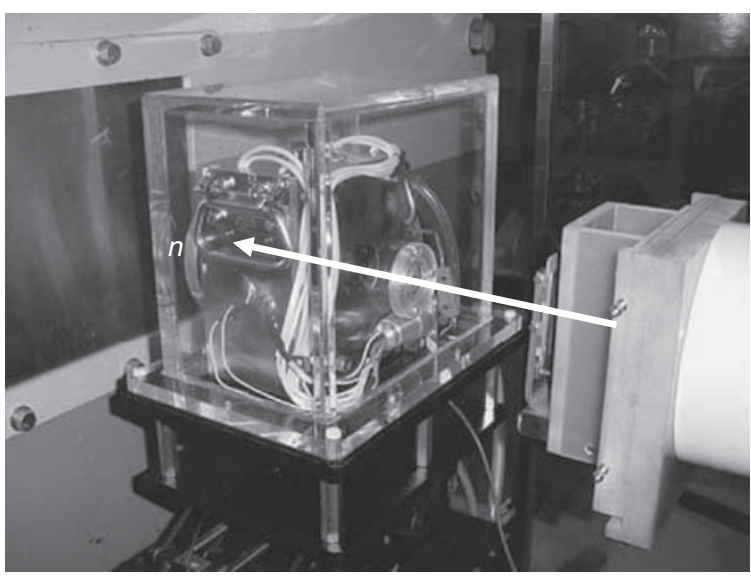

Pressure cell

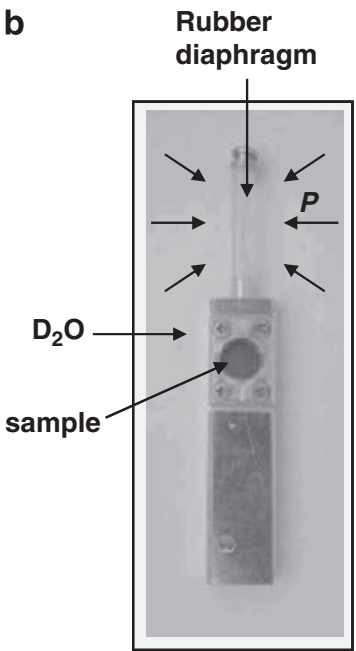

Inner cell

Figure 8 (a) Photographs of the pressure cell installed on SANS-U and (b) the inner cell. $\mathrm{D}_{2} \mathrm{O}$, deuterated water; SANS-U, small-angle neutron scattering instrument owned by University of Tokyo.

b

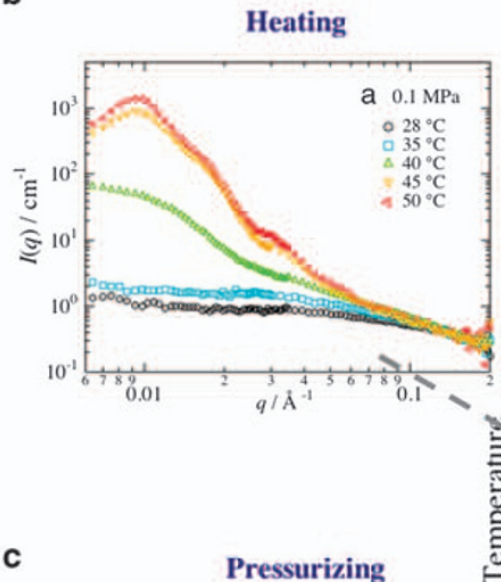

C

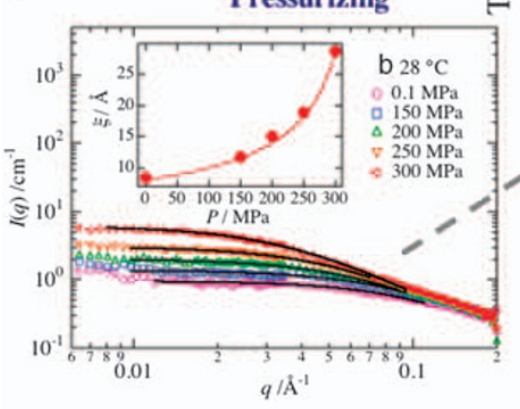

a

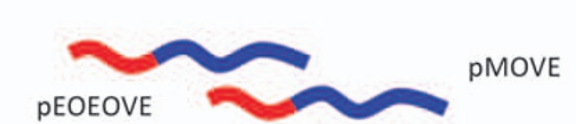

d

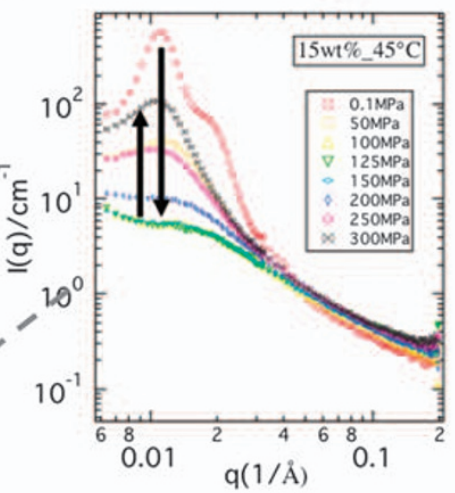

e

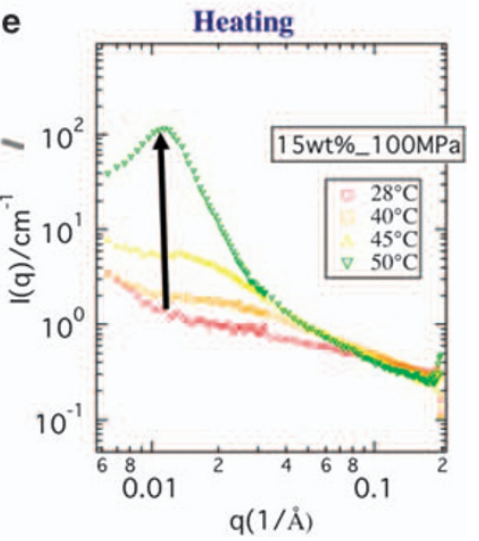

Figure 9 (a) Pressure-temperature phase diagram of poly(2-(2-ethoxy)ethoxyethyl vinyl ether)-block-poly(2-methoxyethyl vinyl ether) (pEOEOVE-pMOVE) in deuterated water; (b-e) the corresponding small-angle neutron scattering patterns.

According to the above discussion, we propose a phase diagram classifying the states of the pEOEOVE-pMOVE aqueous solution as shown in Figure $10 .{ }^{20}$ Here, $\mathrm{A}, \mathrm{B}$ and $\mathrm{S}$ denote the pEOEOVE, pMOVE and the solvent $\left(\mathrm{D}_{2} \mathrm{O}\right)$, respectively, and $T_{\mathrm{A}}$ is the MiST temperature. In region $\mathrm{I}\left(T<T_{\mathrm{A}}\right)$, the system is a homogeneous solution. At low pressures $\left(P<P_{0}\right)$, the system undergoes either a MiST at $T_{\mathrm{A}}$ (region II) or a MaST (precipitation) at $T_{\mathrm{B}}$ (region III) by increasing temperature. Each region is further divided into the strongly hydrophobic solvated region $\left(P<P_{0}\right)$ and the weakly hydrophobic solvated region $\left(P>P_{0}\right)$. In the strongly hydrophobic solvated region, hydrophobic solvation is dominant and $\Delta V_{\mathrm{m}}$ is negative. On the other hand, the weakly hydrophobic solvated region is characterized by $\Delta V_{\mathrm{m}}>0$, resulting in the change in the slope of the $T_{\mathrm{A}}$ curve from positive to negative (from region I to $\mathrm{I}^{\prime}$ or region II to $\mathrm{II}^{\prime}$ ) at 


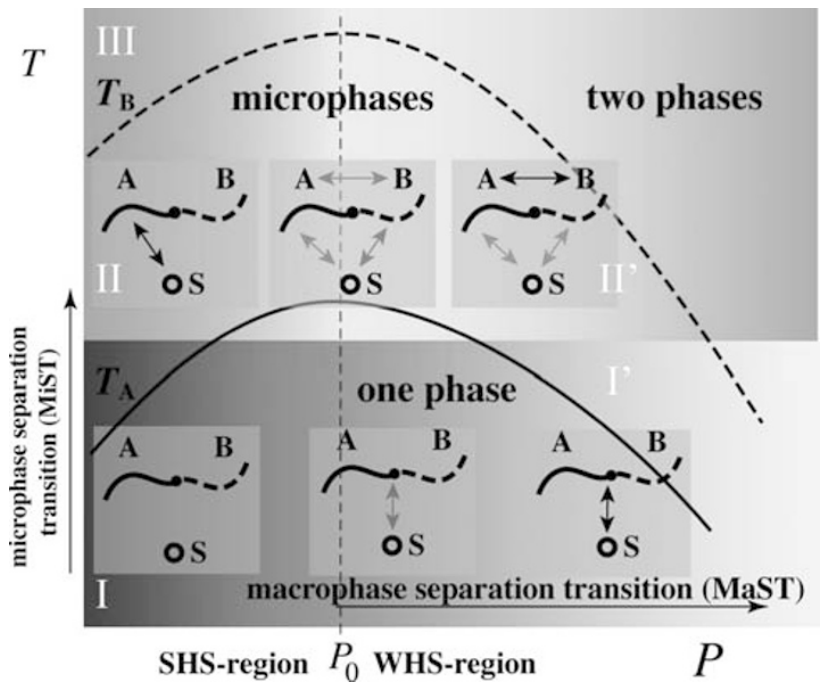

Figure 10 Schematic representation of phase diagram classifying the states of the pEOEOVE-pMOVE solution to characteristic regions. SHS, strongly hydrophobic solvated region; WHS, weakly hydrophobic solvated region.

$P>P_{0}$. From region $\mathrm{I}$ to $\mathrm{I}^{\prime}$, the concentration fluctuations become large, resulting in a macrophase separation at the spinodal pressure, $P_{\text {sp. }}$. The pressure dependence in region II near $T=T_{\mathrm{A}}\left(P_{0}\right)$ is characterized by a reentrant MiST, as revealed by SANS (Figure $9 \mathrm{~d}$ ). Note that the structure in region II' is not highly developed compared with that in region II. This may suggest that the Flory-Huggins interaction parameter $\chi$ in region II' $^{\prime}$ is not as large as that at the symmetric pressure in region II and is a weaker function of temperature.

\section{HIGH-PERFORMANCE POLYMER GELS}

Polymer gels have been believed to be soft and fragile, which is why polymer gels have never been used as structural materials. In the twenty-first century, new types of polymer gels with extraordinarily strong properties have been discovered. Okumura and Ito ${ }^{63}$ developed a new class of gels with movable crosslinks. This gel, called a slide-ring gel (SR gel), is the realization of the concept of a sliding gel or slip-link network. ${ }^{64,65}$ The SR gel can be highly stretched ( $\sim 24$ times) and highly swollen $\left(24 \times 10^{3}\right.$ times). Haraguchi and Takeshita ${ }^{66}$ developed a remarkable gel called a nanocomposite gel (NC gel). Gong et al. ${ }^{67}$ reported double-network gels with high mechanical strength. Sakai et al. ${ }^{68}$ prepared a polymer network by cross-end coupling of two types of four-arm poly(ethylene glycol) (hereafter referred to as TetraPEG gels). All these gels have advanced mechanical properties, such as high deformability and toughness.

Figure 11 shows schematic illustrations of (a) conventional gels, (b) SR gels, (c) NC gels and (d) Tetra-PEG gels. As shown here, the structures of these gels are completely different from each other. The strategies for designing tough gels are completely different as well. In SR gels, movable crosslinkers are introduced to equalize local stress in a polymer network. Novel properties were anticipated by the hybridization of polymer and inorganic materials (NC gels) in light of the success of advanced materials made of tough clay-polymer NCs with high modulus and heat resistance. ${ }^{69,70}$ Tetra-PEG gels represent the realization of ideal polymer networks free of entanglements and defects. Although these gels have been prepared and their physical properties investigated, ${ }^{66,71-73}$ their microscopic structure has not yet been elucidated. We carried out a series of SANS investigations on SR, NC and Tetra-PEG gels in as-prepared ${ }^{74-77}$ and deformed
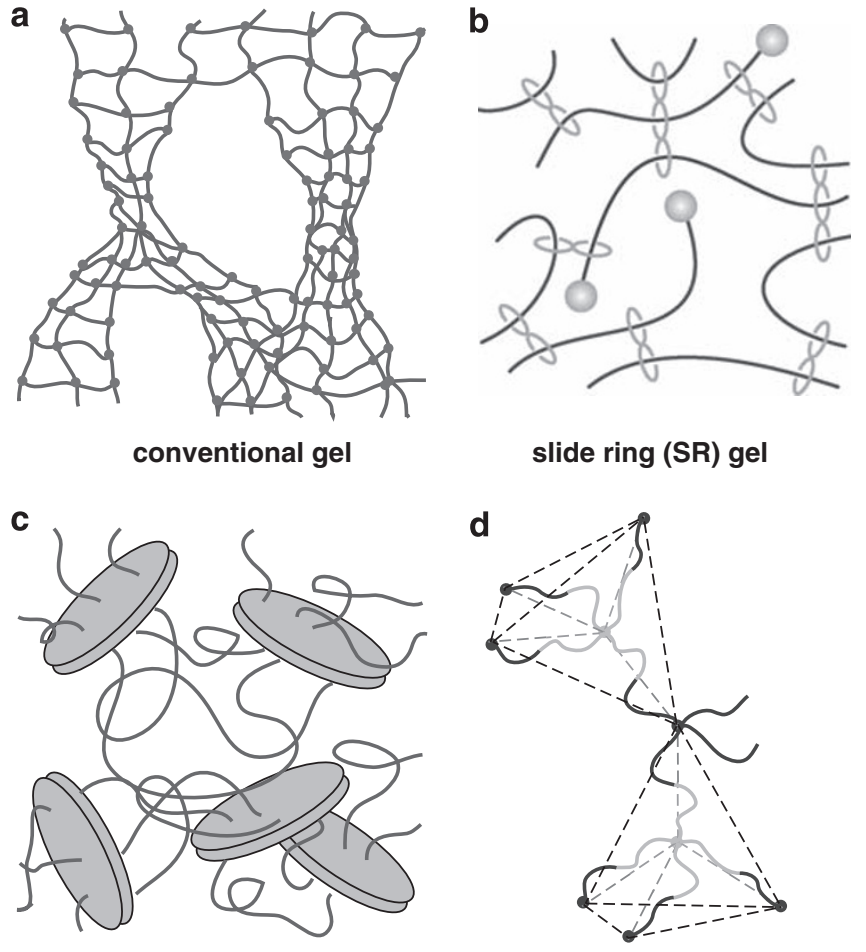

nanocomposite (NC) gel

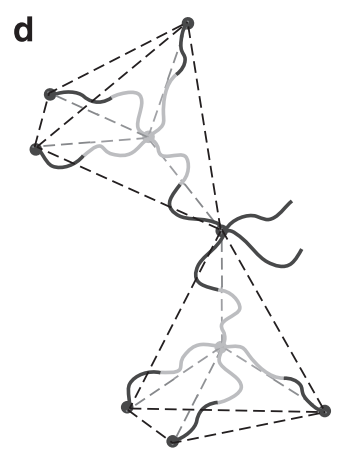

tetra-PEG gel

Figure 11 Schematic illustration of (a) conventional gels, (b) SR gels, (c) NC gels and (d) four-arm poly(ethylene glycol) (Tetra-PEG) gels.

states, ${ }^{78,79}$ and clarified the structure-property relationship as described below.

\section{SR gel: Normal-to-abnormal butterfly transition}

Karino et al. ${ }^{80}$ carried out SANS experiments on poly(ethylene glycol) (PEG), polyrotaxane (PR) solution and SR gels at several crosslink concentrations. It is important to note that in these experiments, both SR and PR gels consist of PEG chains and cyclodextrin; moreover, the former was crosslinked and the latter was not. Hence, a comparison of SANS results among PEG, PR and SR gels was quite interesting. Figure 12 shows SANS results for PEG, PR and SR gels in NaODaq. The SANS curve for PEG (crosses) is a typical scattering function for a flexible polymer solution in a semidilute regime and is well represented by an OZ function (dotted line). The SANS curve for PR solution is a power-law function with $I(q) \sim q^{-1}$, indicating a rod-like conformation. This means that threading a PEG chain to cyclodextrin molecules results in the stiffening of PEG chains. Next, a comparison of SR gels with different crosslink concentrations, $C_{\mathrm{x}}(0.7-3.0 \mathrm{wt} \%)$, disclosed an interesting feature of SR gels. The SANS intensity decreased with increasing $C_{\mathrm{x}}$ from 0.7 (CX07) to $2.0 \mathrm{wt} \%$ (CX20), whereas it increased by further increasing $C_{\mathrm{x}}$ from 2.0 to $3.0 \mathrm{wt} \%$ (CX30). This suggests that crosslinking with SR lowers osmotic compressibility, resulting in a decrease in the scattering intensity, whereas further crosslinking leads to an increase in inhomogeneities. Thus, SR gels undergo a reentrant transition in inhomogeneities by increasing $C_{\mathrm{x}}$.

Figure 13 shows the SANS result of deformed SR gels, that is, CX07 $\left(C_{\mathrm{x}}=0.7 \%\right.$; top $)$ and $\mathrm{CX} 20\left(C_{\mathrm{x}}=2.0 \%\right.$; bottom $) .{ }^{78}$ The stretching direction was horizontal. Interestingly, CX07 showed a normal butterfly pattern, that is, a prolate scattering pattern elongated perpendicular 
to the stretching direction. Because conventional gels exhibit an abnormal butterfly pattern, this observation was quite interesting. This pattern indicates that the contribution of crosslinks, in other words, inhomogeneities to structural change, by deformation is negligible. On the other hand, an abnormal butterfly pattern was observed in CX20 (see the bottom figure). This suggests that normalto-abnormal butterfly transition takes place in SR gels by simply increasing $C_{\mathrm{x}}$.

Figure 14 schematically shows the swelling behavior of conventional gels (left) and SR gels (right). As discussed in Figure 3, the swelling of conventional gels leads to an enhancement of inhomogeneities. On the other hand, such increase in inhomogeneities by swelling is strongly suppressed in SR gels because of movable crosslinkers. However, the effect of movable crosslinks is restricted in low crosslinker concentrations (for example, $C_{\mathrm{x}}=0.7 \%$ ). For larger $C_{\mathrm{x}}$ (for example, $C_{\mathrm{x}}=2.0 \%$ ), SR gels function similarly to conventional gels, as discussed in

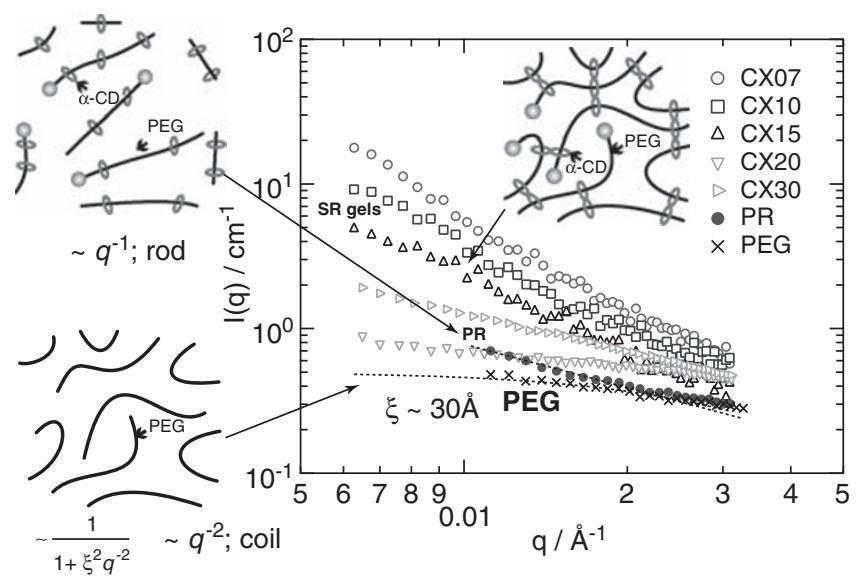

Figure 12 Small-angle neutron scattering (SANS) results for poly(ethylene glycol) (PEG), polyrotaxane (PR) and slide-ring (SR) gels in NaODaq.
Figure 12. Therefore, it is important to maintain the crosslink density as low as possible to functionalize the movable crosslinks.

\section{NC gels: contrast variation SANS}

NC gels are comprised of polymer, clay and water. ${ }^{66} \mathrm{NC}$ gels can be simply prepared by mixing synthetic clay (Laponite) and PNIPA monomer in water and allowing them to polymerize with ammonium persulfate. $^{71,72}$ The mechanical properties can be easily chosen by the composition. ${ }^{81}$ The structure-property relationship was extensively investigated by SANS. ${ }^{74,75,78,82}$ Because NC gels are three-component systems, the scattering intensity function consists of six partial scattering functions, $S_{i j}(q)$ where $i, j=1,2$ and 3 . However, because of the incompressibility assumption (that is, $\sum_{i=1}^{3} S_{i j}(q)=0$ for $j=1$, $2,3)$, they are reduced to three independent partial scattering functions, as schematically shown in Figure 15, and are written as follows (on the basis of the assumption of incompressibility): ${ }^{76}$

$I(q)=\left(\rho_{1}-\rho_{3}\right)^{2} S_{11}(q)+\left(\rho_{2}-\rho_{3}\right)^{2} S_{22}(q)+2\left(\rho_{1}-\rho_{3}\right)\left(\rho_{2}-\rho_{3}\right) S_{12}(q)$

Here, $\rho_{\mathrm{i}}\left(=\sum_{j} b_{j} / v_{i}\right)$ indicates the scattering length density of each component, $i$ ( $i=1,2$ and 3 , or $\mathrm{c}, \mathrm{p}$ and $\mathrm{w}$, respectively), $b_{j}$ is the scattering length of $j$-th atom and $v_{i}$ is the volume of the molecule. $S_{i j}(q)$ s are the partial scattering functions. $S_{11}(q)$ and $S_{22}(q)$ are called the self-terms, representing the self-correlations of component 1 and 2 , respectively. $S_{12}(q)$ is called the crossterm, representing the crosscorrelation between component 1 and 2 . Note that $S_{i j}(q)$ is normalized by the absolute intensity and the scattering length density and has the dimensions of volume. We performed contrast variation SANS (CVSANS) experiments by tuning the scattering length density of component $3, \rho_{3}$, (that is, $\rho_{\mathrm{w}}$ ) in order to decompose the scattering intensities into partial scattering functions as below:

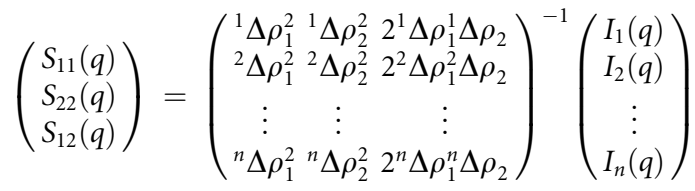
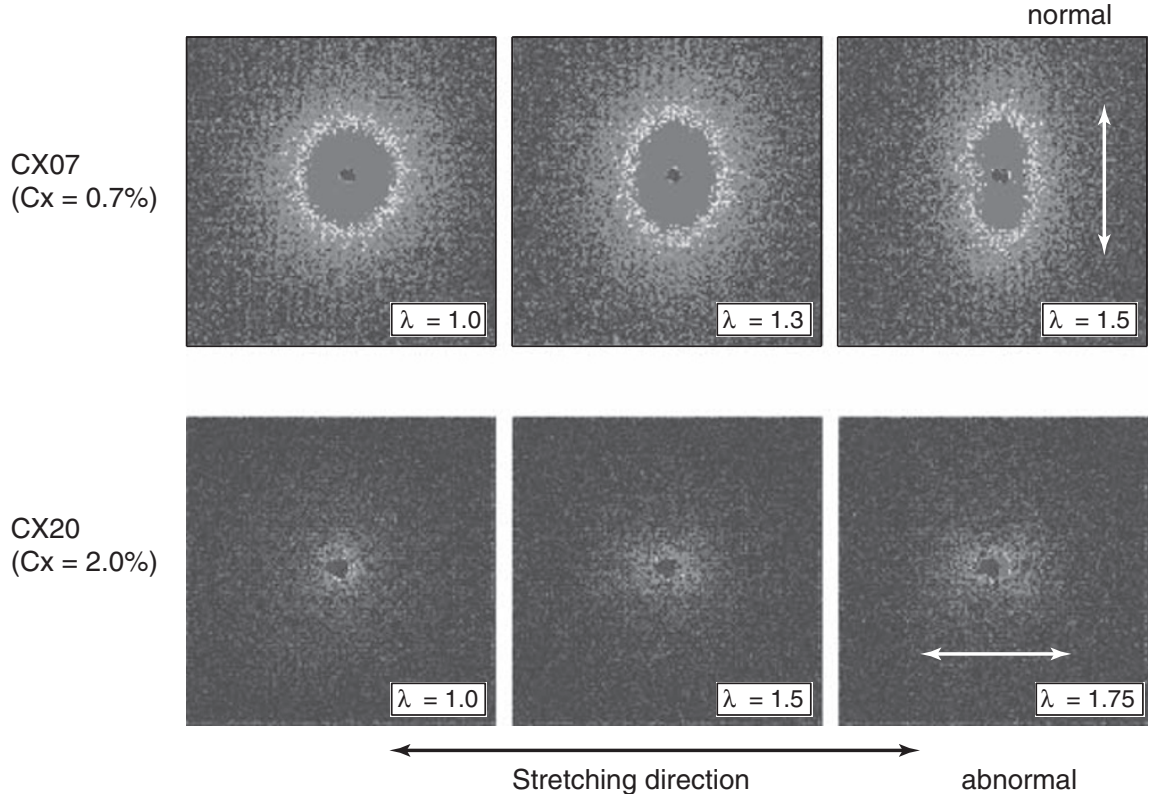

Figure 13 Two-dimensional (2D) small-angle neutron scattering (SANS) patterns of slide-ring (SR) gels showing normal-to-abnormal butterfly transition. $C_{X}$, crosslink concentrations. 

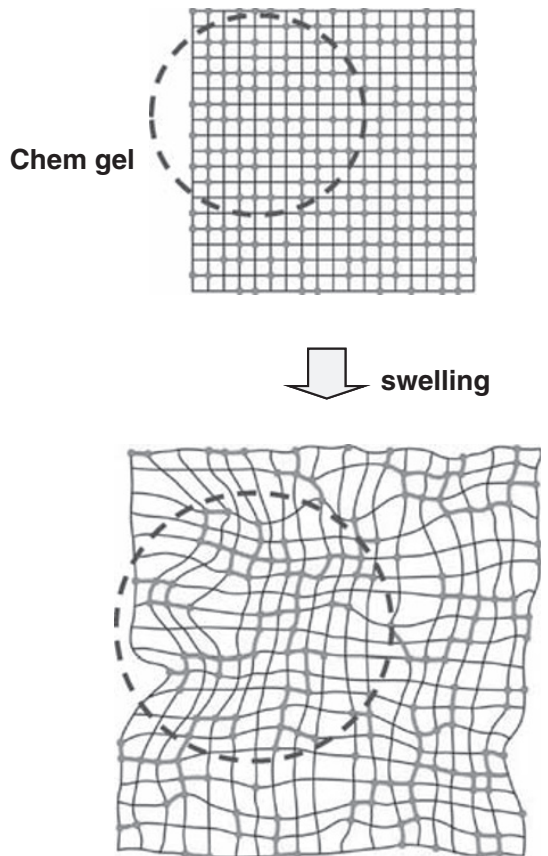

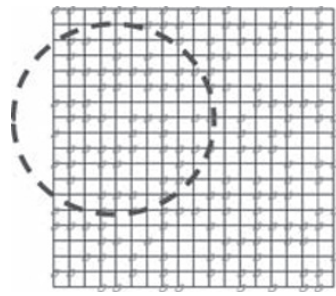

SR gel

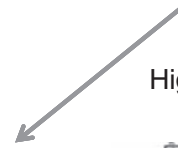

High $C_{x}$
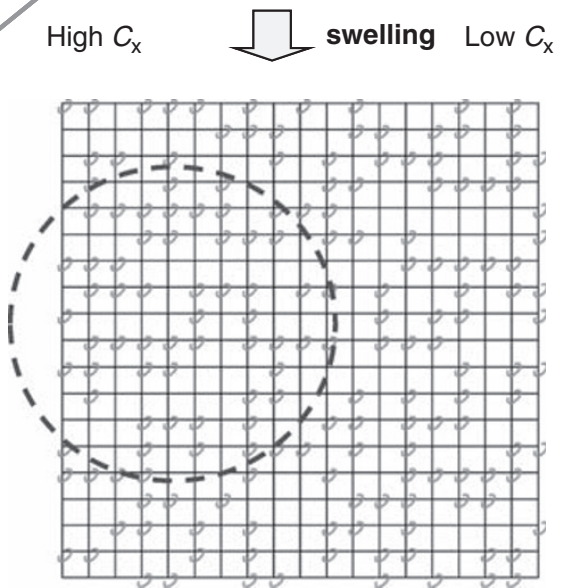

Figure 14 Schematic illustration of swelling behaviors of conventional gels (left) and slide-ring (SR) gels (right). $C_{X}$, crosslink concentrations.

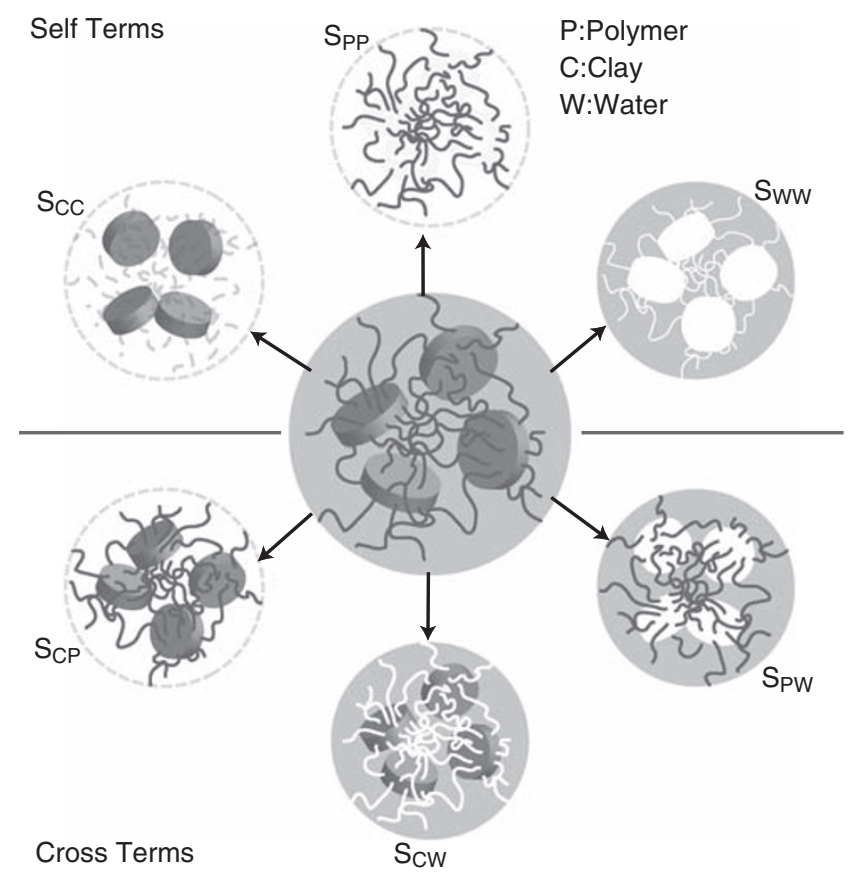

Figure 15 Schematic illustration of contrast variation and partial scattering functions for nanocomposite (NC) gels.

where ${ }^{n} \Delta \rho_{i} \equiv \rho_{i}-^{n} \rho_{3}$ is the difference of the scattering length densities between component $i$ and 3 (that is, water) of the $n$-th measurement. Here, the scattering length density of water was varied by changing the $\mathrm{D} / \mathrm{H}$ fraction, ${ }^{n} \rho_{3}$. The transposed matrix of $(3 \times n)$ contrast matrix,
${ }^{\mathrm{T}}\left({ }^{3 \times n} \Delta \rho\right)$, which fulfills the condition ${ }^{\mathrm{T}}\left({ }^{3 \times n} \Delta \rho\right)\left({ }^{3 \times n} \Delta \rho\right)=\mathbf{E}$, can be obtained by singular value decomposition, where $\mathbf{E}$ is the identity matrix. A detailed description of the procedure can be found elsewhere. ${ }^{83}$ Analysis of $S_{\mathrm{PP}}(q), S_{\mathrm{CC}}(q)$ and $S_{\mathrm{CP}}(q)$ indicates that (i) each clay platelet with $\sim 150-\AA$ radius and $\sim 10-\AA$ thickness is surrounded by polymer layers with $\sim 200$ - $\AA$ radius and $\sim 30-\AA$ thickness, including the clay platelet, (ii) the volume fraction of the polymer layer per clay platelet is independent of the concentrations and (iii) the correlation length of the network polymer decreases with increasing clay concentration. ${ }^{76}$

Because NC gels are very tough and can be stretched more than 10 times their original length, SANS studies of NC gels are much easier than of conventional PNIPA gels. Figure 16 shows a laboratory-made stretching device on which NC15 $\left(0.15 \mathrm{~mol}\left(114.5 \mathrm{gl}^{-1}\right)\right.$ of clay and $1 \mathrm{~mol}\left(113 \mathrm{gl}^{-1}\right)$ of PNIPA) gels are mounted. Here, the sample size was $4 \mathrm{~cm}$ long (including chucking parts), $3 \mathrm{~cm}$ wide and $4 \mathrm{~mm}$ thick when it was crimped. CV-SANS was applied to investigate the deformation mechanism of $\mathrm{NC} 15$ gels. As shown in Figure 17, anisotropic SANS functions were obtained at various stretching ratios, and $\lambda$ values up to $\lambda=9$ were decomposed into three partial structure factors, $S_{\mathrm{CC}}(q), S_{\mathrm{PP}}(q)$ and $S_{\mathrm{CP}}(q)$. Here, $q_{\text {para }}$ and $q_{\text {perp }}$ are the magnitude of the scattering vectors along and perpendicular to the stretching directions, respectively. $S_{\mathrm{CC}}(q)$ and $S_{\mathrm{PP}}(q)$ indicate that clay platelets are oriented along the stretching direction, and the polymer chains are deformed in the stretching direction. $S_{\mathrm{CP}}(q)$, only available by CV-SANS, suggests that a polymerenriched layer is formed on the clay surface, which serves as a reservoir of large extensibility of NC gels over $1000 \%$ strain. CV-SANS was also applied to study microphase separation of NC gels by heating. ${ }^{84}$ Interestingly, $S_{\mathrm{CC}}(q)$ is not affected by microphase separation, whereas $S_{\mathrm{PP}}(q)$ increased and showed a scattering maximum, as was observed in PNIPA/AAc gels. 


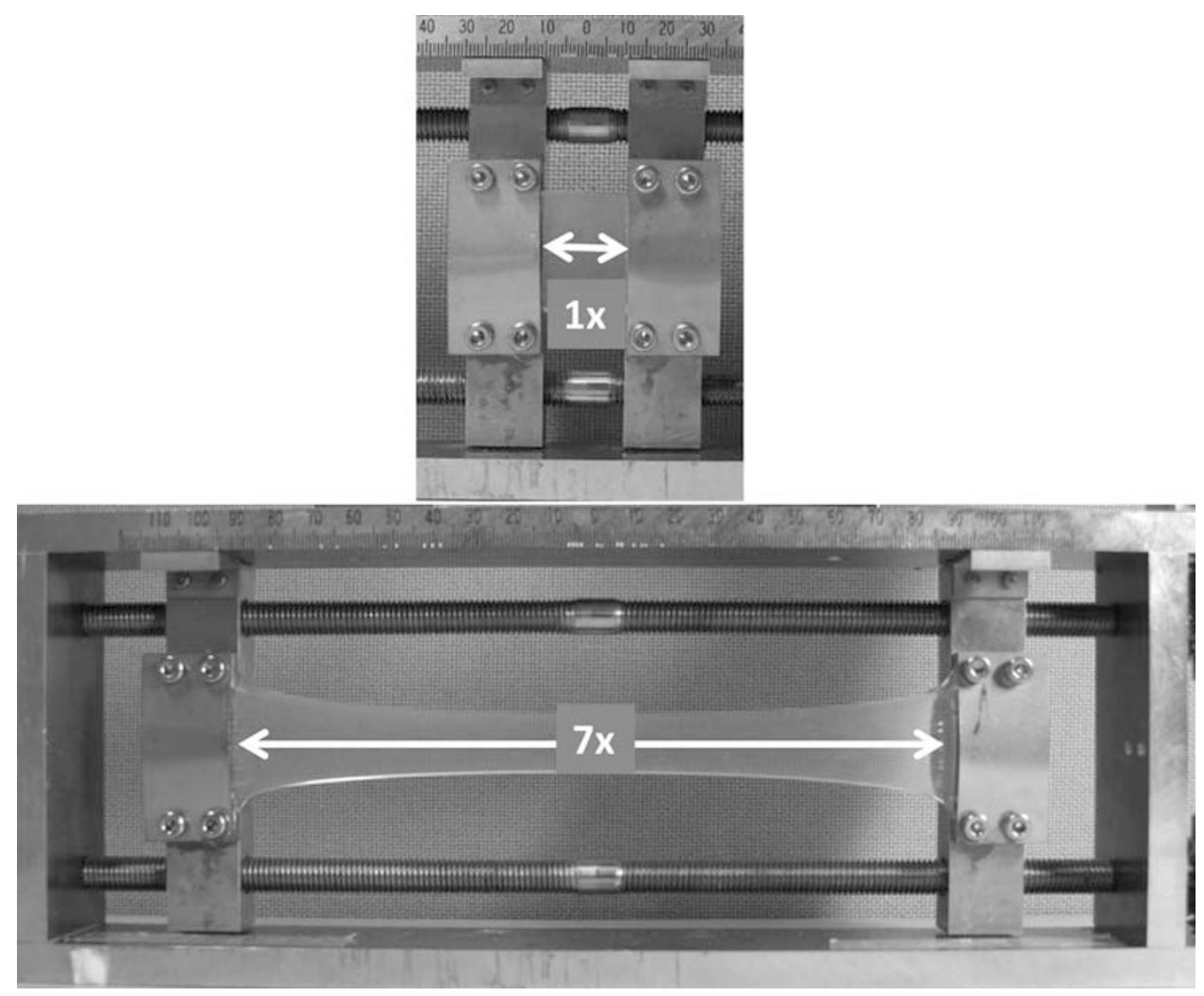

Figure 16 Laboratory-made stretching device for small-angle neutron scattering experiments and NC gels at $\lambda=1$ (top) and = 7 (bottom).

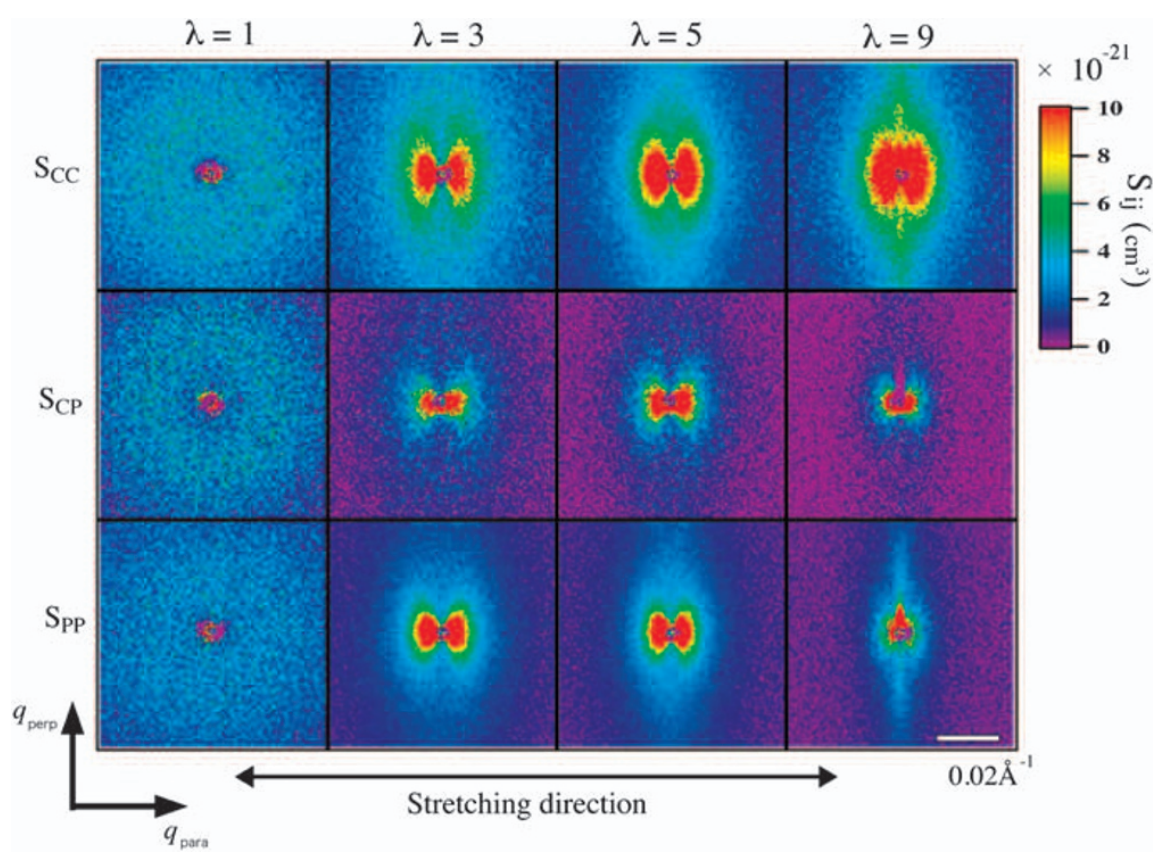

Figure 17 Two-dimensional (2D) small-angle neutron scattering (SANS) patterns of nanocomposite (NC) gels decomposed by contrast variation.

\section{Tetra-PEG gels: ideal polymer network}

Tetra-PEG gels are a new class of biocompatible, easily made and highstrength hydrogels. PEG gel consists of two kinds of Tetra-PEG macromers of the same size: tetraamine-terminated PEG (TAPEG) and tetra-NHS-glutarate-terminated PEG (TNPEG). Here, NHS represents $N$-hydroxysuccinimide. By mixing TAPEG and TNPEG aqueous solutions, Tetra-PEG gel can be instantaneously formed, not only in the laboratory but also in vivo. Tetra-PEG gels are prepared 
Tetra-PEG gel: dried, stretched, re-swollen

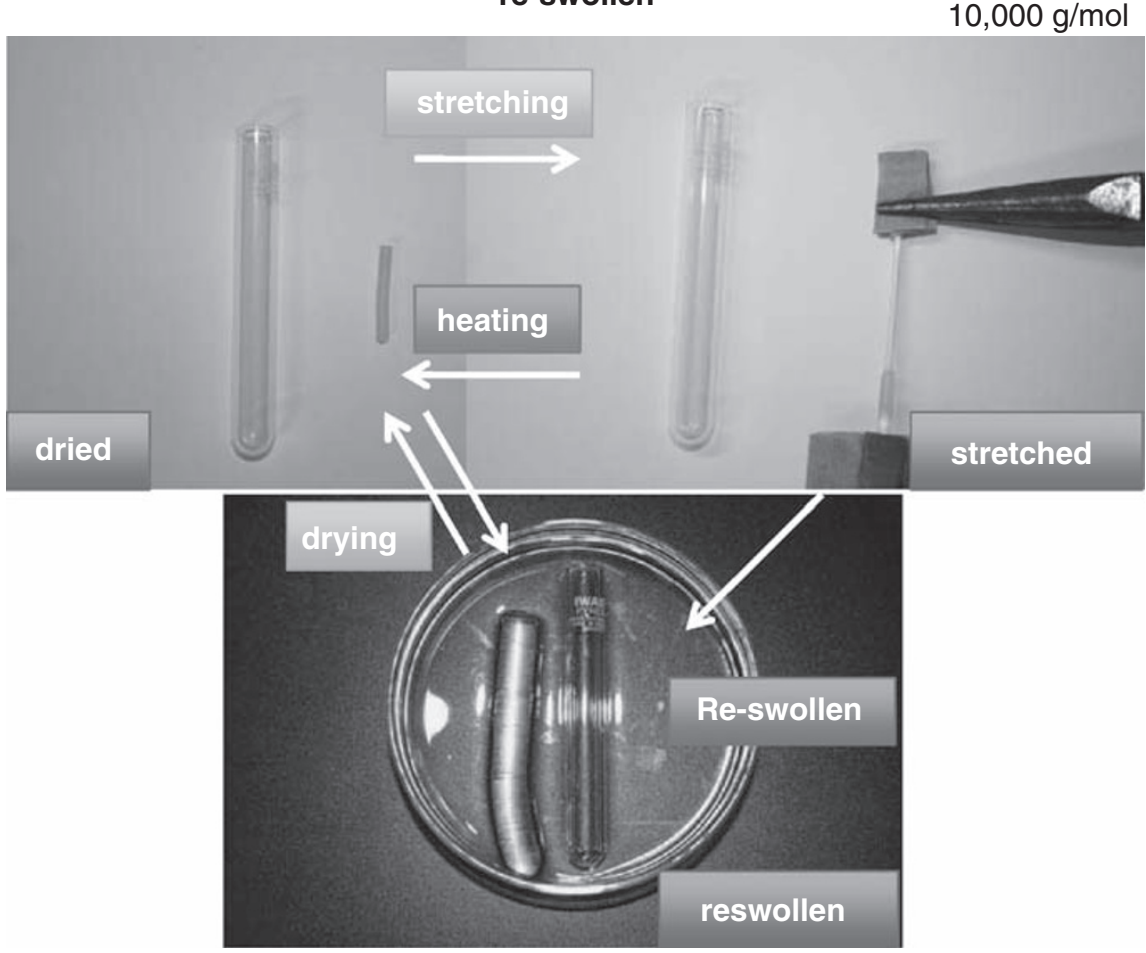

Figure 18 Pictures of Tetra-PEG-10K. First, Tetra-PEG gels were prepared in a test tube, which can be dried to a small rod (top left) and stretched with neck deformation (top right). Tetra-PEG, four-arm poly(ethylene glycol).

by cross-end coupling two kinds of Tetra-PEG macromers with different functional groups at the ends, that is, amine group and succinimidyl ester group, respectively; therefore, the coupling reaction occurs exclusively between PEG chains carrying different functional groups. $^{85}$ The mechanical properties of Tetra-PEG gels change depending on the molecular weight of the parent macromers. ${ }^{86}$ Tetra-PEG-5K is rather brittle and cannot be highly stretched; $-5 \mathrm{~K}$ denotes the molecular weight of the parent macromer. On the other hand, Tetra-PEG-10K, Tetra-PEG-20K and Tetra-PEG-40K have notable mechanical strength and deformability. Figure 18 shows a set of pictures of Tetra-PEG-10K. First, Tetra-PEG-10K gels were prepared in a test tube, and then they were dried to a small rod (top left) and stretched with neck deformation (top right). Nondeformed dried (left) or as-prepared Tetra-PEG gel (bottom) could be recovered from the stretched state by heating (from top-right to left) or immersing in water (from top-left to bottom). This figure demonstrates the resistance of Tetra-PEG gel against heating, swelling and stretching. Evaluation of topological defects, ${ }^{87}$ gelation kinetics of Tetra-PEG gels ${ }^{85}$ and the effect of module size on the network structure of Tetra-PEG gel ${ }^{88}$ are extensively investigated by Sakai and co-workers.

Figure 19 shows a series of SANS functions of Tetra-PEG-5K, -10K, $-20 \mathrm{~K}$ and $-40 \mathrm{~K}$ gels, prepared at different polymer concentrations, $\phi_{0}$. The solid lines are the curve fits with an OZ function (Equation 4). Note that all the SANS curves were successfully fitted with an OZ function, except for Tetra-PEG-5K gels prepared at low polymer concentrations. It is well known that polymer gels usually exhibit a strong upturn in the low- $q$ region because of the presence of inhomogeneities. ${ }^{16,89}$ Even in the case of 'ideal polymer networks' made by end-linking of telechelic polymer chains, significant inhomogeneities are reported. ${ }^{90,91}$ Hence, the observed result for Tetra-PEG gels is quite surprising.

Figure 20 is a schematic representation of the network structure of Tetra-PEG gels. Because of cross-end coupling of the TAPEG and TNPEG chains, Tetra-PEG networks are formed in such a way that TAPEG and TNPEG macromers are alternatively arranged in the space. Hence, Tetra-PEG gels are 'ideal' polymer networks consisting of elastic blobs without any entanglements. As a matter of fact, Sakai and coworkers ${ }^{87,92}$ reported that Tetra-PEG does not have a noticeable degree of entanglement. Figure 21 shows a series of stroboscopic photos of a (a) Tetra-PEG ball and (b) commercial power ball. When they were dropped from a certain distance above a table, both balls bounced well, and the bounce height of the Tetra-PEG ball was comparable to that of the power ball with a repulsion coefficient of 0.84 . It should be noted here that the polymer concentration was only $15 \%$. Such high elasticity was confirmed by a very low loss tangent on the order of $10^{-3}$, as was measured by a viscoelastic measurement.

Figure 22 is a master plot of SANS curves for Tetra-PEG gels with various molecular weights, that is, $-5 \mathrm{~K},-10 \mathrm{~K},-20 \mathrm{~K}$ and $-40 \mathrm{~K}$, prepared at various polymer concentrations, $\phi_{0} \cdot{ }^{93}$ All of the SANS intensity functions could be superimposed with the reduced variables $I(q)$ / $\left(\phi_{0} \xi^{2}\right)$ and $q \xi$. For $q \xi \geqslant 10^{-1}$, the scattering functions fall onto a single master curve given by an Ornstein-Zernike function: ${ }^{86,93}$

$$
\frac{I(q)}{\phi_{0} \xi^{2}} \sim \frac{1}{1+(\xi q)^{2}}
$$

The cross-symbols are the scattering intensity data for linear PEG chains in $\mathrm{D}_{2} \mathrm{O}$ observed by Hammouda et al. ${ }^{94,95}$ and are assigned to clustering of PEG chains by the association of end groups. Hence, it is deduced that the strong upturn in $I(q) /\left(\phi_{0} \xi^{2}\right)$ at low- $q \xi$ for 


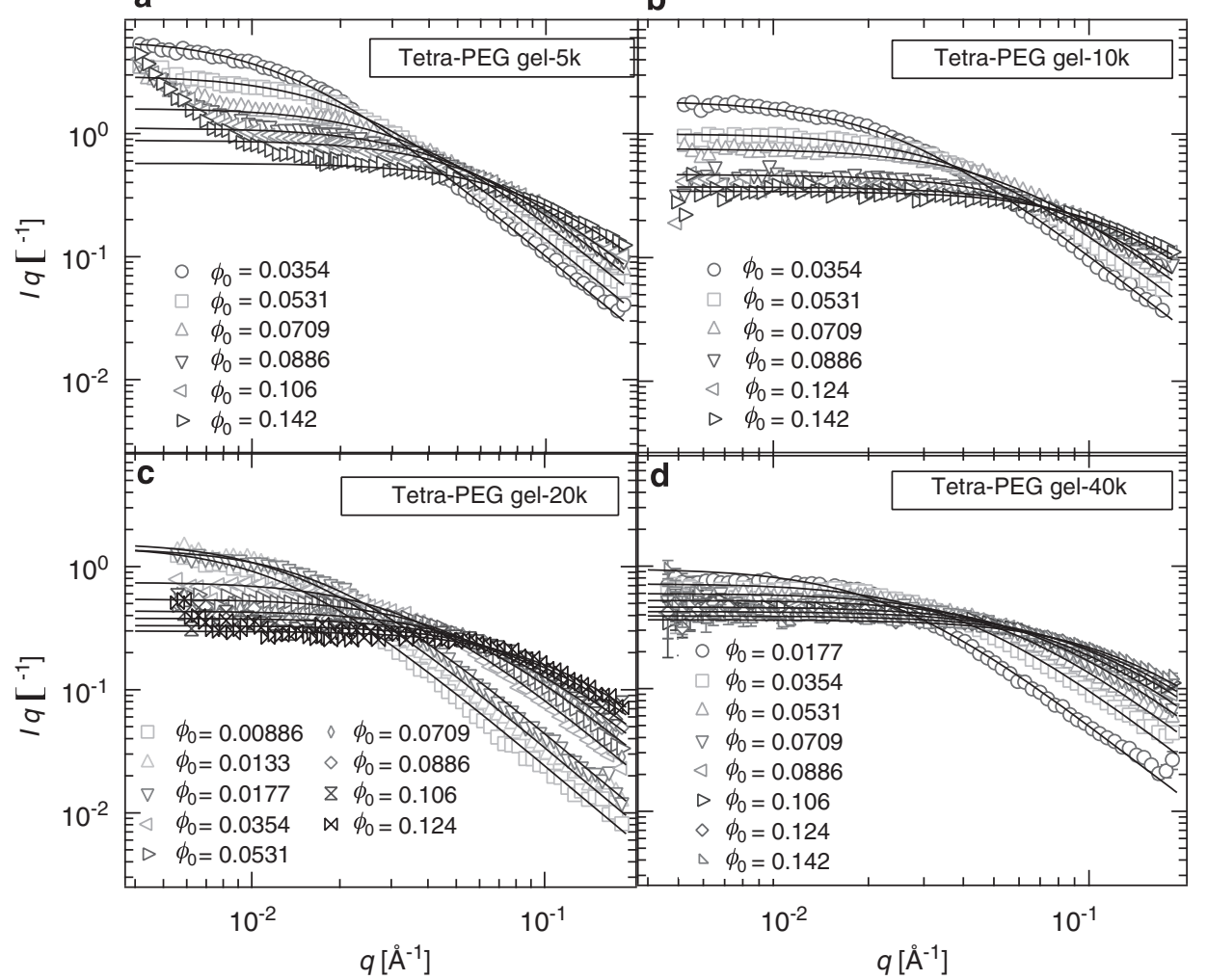

Figure 19 Concentration and $\mathrm{M}_{\mathrm{w}}$ dependence of small-angle neutron scattering (SANS) intensity functions of four-arm poly(ethylene glycol) (Tetra-PEG) gels with $-5 \mathrm{~K},-10 \mathrm{~K},-20 \mathrm{~K}$ and $-40 \mathrm{~K}$.

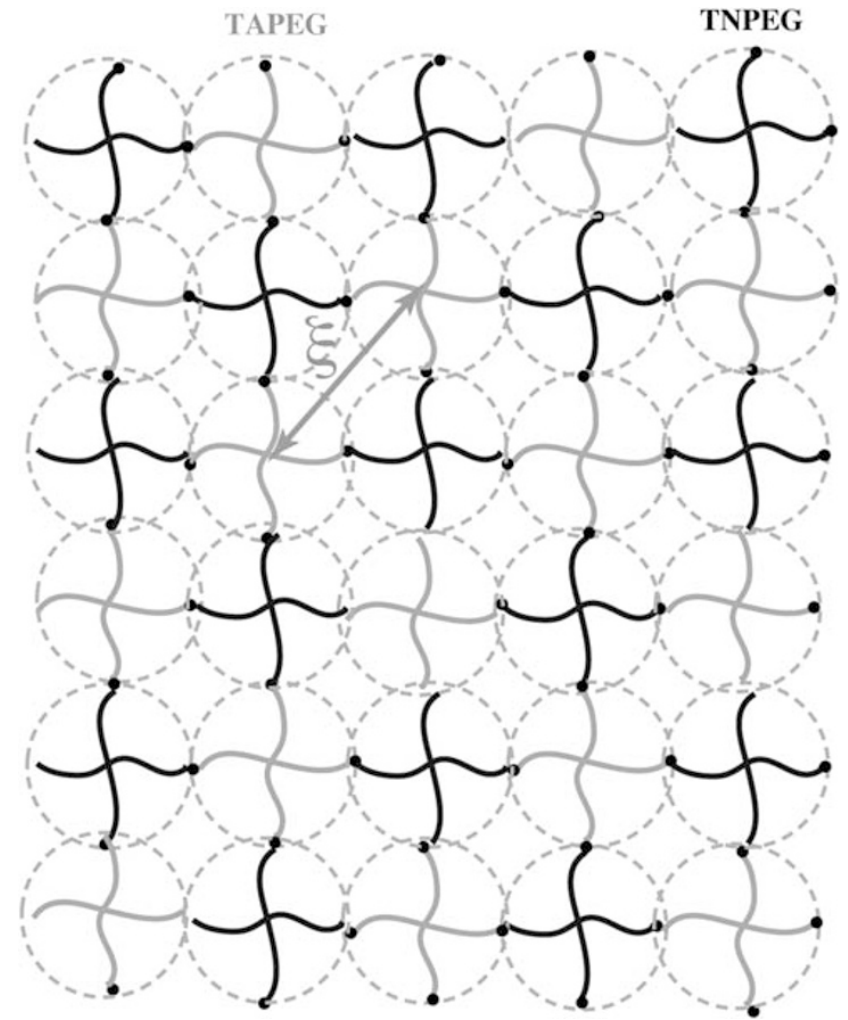

Figure 20 Schematic illustration of the network structure of four-arm poly(ethylene glycol) (Tetra-PEG) gels. TAPEG, tetraamine-terminated PEG; TNPEG, tetra- $N$-hydroxysuccinimide-glutarate-terminated PEG.
Tetra-PEG-5K is ascribed to the clustering of PEG chains. The upturn for Tetra-PEG-10K, $-20 \mathrm{~K}$ and $-40 \mathrm{~K}$ has not yet been assigned and is now under investigation.

\section{Deformation mechanism of super-tough gels}

In the preceding sections, various types of deformation mechanisms were discussed for SR gels and NC gels. In addition to these supertough gels, Tetra-PEG gels are also highly stretchable. It has recently been confirmed that the degree of retraction is even better than that of any other super gels or commercial rubber rings. ${ }^{92}$ SANS of deformed Tetra-PEG gels showed invariance of 2D-SANS patterns on uniaxial stretching; that is, inhomogeneities do not appear even by stretching. This is another characteristic feature of Tetra-PEG gels.

We can summarize the deformation mechanisms of super-tough gels in Figure 23. Conventional gels made by chemical reaction of a mixture of monomer and crosslinker have a high degree of inhomogeneities in their network structure, namely, dangling chains, loops and crosslinker-rich and -poor regions. This is why conventional gels are brittle and easily broken by stretching only a few tens of percent. ${ }^{78}$ The 2D-SANS patterns exhibit abnormal butterfly patterns, indicating the presence of an inhomogeneous network structure (see for example Figure 6). ${ }^{46}$ SR gels are unique in their network structure because of their movable crosslinkers. When an SR gel is stretched, the crosslinks are believed to slide along the polymer chain so as to minimize the local strain. This mechanism guarantees high elasticity and high deformability of SR gels. The type of 2D-SANS patterns depends on the crosslinker concentration, $C_{\mathrm{x}}$. If $C_{\mathrm{x}}$ is low enough, the SR gel behaves as a 'SR' gel, and exhibits a 'normal' butterfly pattern free of inhomogeneities. However, by increasing $C_{\mathrm{x}}$, SR gels change to 'non-SR' gels, and their 2D-SANS patterns become abnormal. In the 

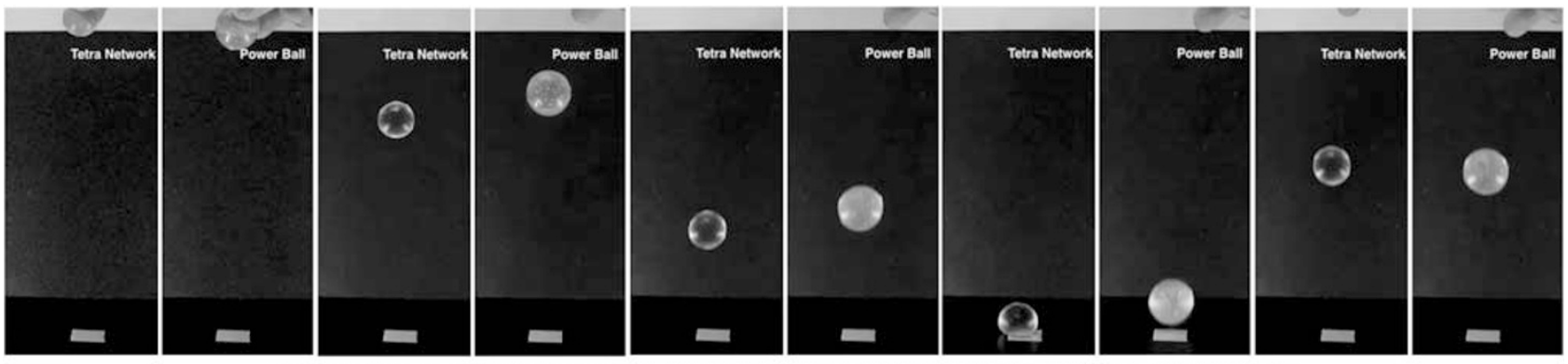

strobo-photos of ball drop experiment; (a) tetra-PEG gel (left), (b) power ball (right)

Figure 21 Five sets of stroboscopic photos of (a) a four-arm poly(ethylene glycol) (Tetra-PEG) ball (left) and (b) a commercial power ball (right).

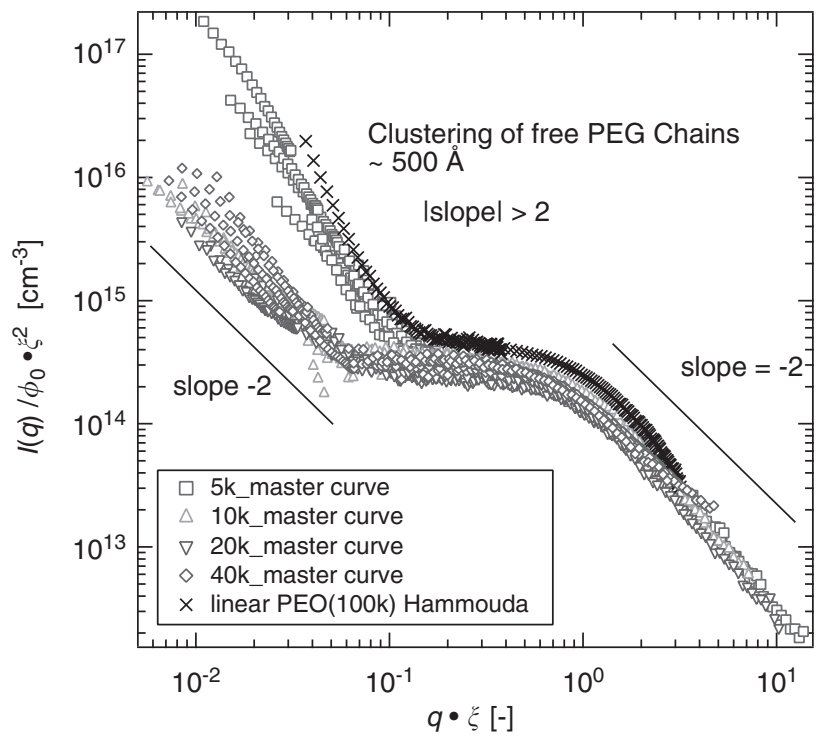

Figure 22 Master plots of small-angle neutron scattering (SANS) curves for four-arm poly(ethylene glycol) (Tetra-PEG) gels with various molecular weights, $-5 \mathrm{~K},-10 \mathrm{~K},-20 \mathrm{~K}$ and $-40 \mathrm{~K}$, and concentrations, $\phi_{0}$, during preparation. The crosses show the SANS curve of linear poly(ethylene glycol) (PEG) chains in deuterated water $\left(\mathrm{D}_{2} \mathrm{O}\right)$.

case of NC gels, the deformation mechanism is more complicated than in other types of gels, because both clay and polymer chains are involved in deformation. The high elasticity and high deformability of NC gels are acquired by the strong affinity between clay and PNIPA. It is believed that amino groups of PNIPA are strongly attracted to the functional groups of clay. ${ }^{69,70}$ As a result, many PNIPA chains are strongly bound to the surface of clay platelets (See Figure 11c), which function as 'wire of reel' or 'reservoir of polymer chains' in NC gels. The clay platelets are 'plane' crosslinkers, which effectively increase the crosslinking density. Furthermore, it is of importance that the molecular weight of PNIPA connecting clay platelets is more than a few million. ${ }^{96,97}$ The 2D-SANS patterns for deformed NC gels exhibit no inhomogeneity at $\lambda=1$, but do show unique patterns of clay orientation/rearrangement in clay scattering and a 'normal' butterfly pattern in polymer scattering (Figure 17). Tetra-PEG gels consist of an elastic blob with no defects or entanglements. Thus, its deformation mechanism seems to be much simpler. An assembly of elastic blobs assures high elasticity and high deformability of Tetra-PEG gels. Interestingly, 2D-SANS patterns do not exhibit any anisotropy and maintain isotropic scattering. ${ }^{92}$ Further investigations to understand this unique scattering behavior of Tetra-PEG gels are in progress.

\section{CONCLUDING REMARKS}

Recent developments in SANS investigations on polymer gels and environment-sensitive block copolymers are reviewed. These developments owe much to (1) the physical approach in understanding fascinating phenomena in polymer gels, such as VPT and critical phenomena, (2) the phenomenological and theoretical understanding of gel inhomogeneities, (3) the discovery of novel super-tough gels and (4) the advancement of methodologies for the quantitative and precise analyses of SANS intensity functions, such as the contrast variation method and incoherent intensity evaluation. ${ }^{98}$ The fruitful outcomes of SANS investigations include molecular-level understanding of (1) the VPT and microphase separation of environmentsensitive polymer gels, (2) the relationship between inhomogeneities and molecular and environmental parameters of gels, (3) the roles of hydrophobic interactions in aqueous media and (4) the deformation mechanisms of various types of super-tough gels.

In connection with these achievements in the structural investigations of polymer gels, we would like to propose the paradigm 'realization of ideal polymer network'. In the long history of polymer networks, which began with the discovery of vulcanization (in the nineteenth century), we have been pursuing 'ideal polymer network' with $100 \%$ crosslinking, without defects, such as dangling chains or loops, and with no entanglements. Figure 24 shows the history of polymer networks. At the beginning of the twentieth century, bakelite was discovered, which is still one of the leading thermoset plastics. Random crosslinking was used to make polymer networks around the 1930s. However, the control of network structure, such as chain length between neighboring crosslinks, was difficult. In the 1970s, model networks were prepared by end-linking monodisperse telechelic polymers. However, analyses using extracted sol fraction, elastic moduli, swelling degree and SANS revealed that the obtained model networks did contain substantial amounts of inhomogeneities. ${ }^{99,100}$ SR gels, discovered in 2001, have changed the concept of what constitutes a polymer network. ${ }^{65}$ Although such a network had been predicted by theoreticians as a 'slip-link model, ${ }^{64}$ its realization was momentous. As a matter of fact, this invention (discovery) was introduced in Nature Materials by Granick and Rubinstein. ${ }^{101}$ The Tetra-PEG gels, if made perfectly without any defects, can be called ideal polymer networks'. Ideal Tetra-PEG gels (or elastomers formed by dehydration) can have advanced mechanical properties with high deformability with no loss tangent and high optical clarity because of the lack of heterogeneities. Tetra-PEGs can be used as molecular sieves with uniform mesh sizes. Hence, ideal polymer networks will establish a 


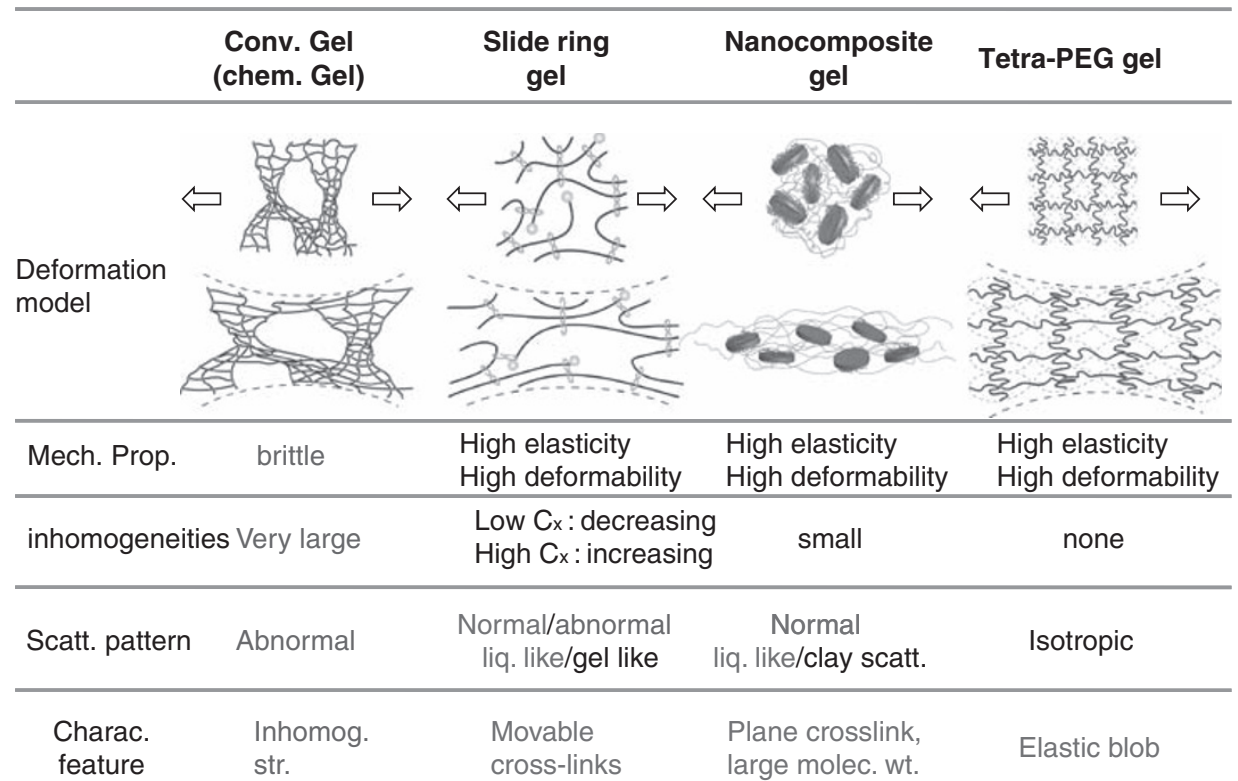

Figure 23 Summary deformation mechanisms of super-tough gels. $C_{X}$, crosslink concentrations; Tetra-PEG, four-arm poly(ethylene glycol).

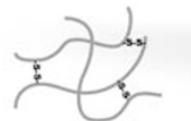

1839 vulcanization

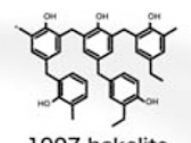

1907 bakelite exploration of "ideal" polymer network

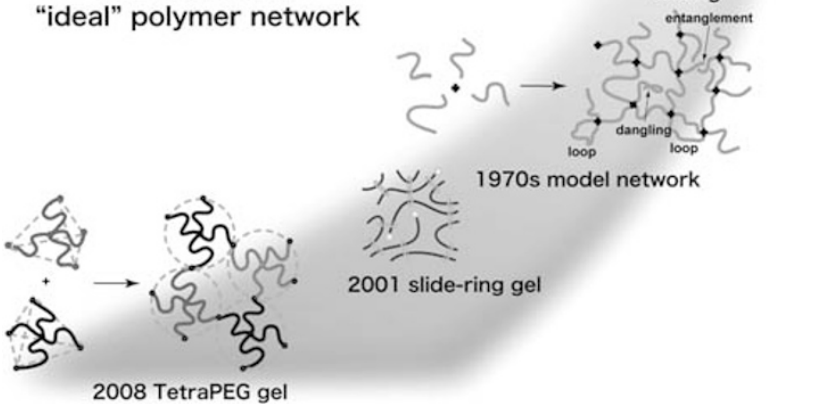

Figure 24 History of polymer networks toward the realization of ideal polymer network. Tetra-PEG, four-arm poly(ethylene glycol).

new paradigm in polymer science and structural characterization, with SANS having an important role.

\section{ACKNOWLEDGEMENTS}

I thank the collaborators, Professors Toyoichi Tanaka, Yitzhak Rabin, Kohzo Ito, Sadahito Aoshima, Dr Sergey Panykov, Dr Fumiyoshi Ikkai, Dr Satoshi Okabe, Dr Irina Nasimova, Dr Kazutoshi Haraguchi, Dr Hitoshi Endo, Dr Takeshi Karino, Dr Noboru Osaka and other colleagues in my laboratory. I also acknowledge the financial support of the Ministry of Education, Science, Sports and Culture, Japan (Grant-in-Aid for Scientific Research (A), 2006-2008, No. 18205025 and 2010-2012, No. 22245018, and for Scientific Research on Priority Areas, 2006-2010, No. 18068004). The SANS experiment was performed with the approval of the Institute for Solid State Physics, The University of Tokyo, at the Japan Atomic Energy Agency, Tokai, Japan.
1 Doty, P. M., Zimm, B. H. \& Stein, R. S. Classical theory of ligbt scattering from solutions-A review. Polym. Bull. 1, 90 (1945).

2 Zimm, B. H. The scattering of light and the radial distribution function of high polymer solutions. J. Chem. Phys. 16, 1093-1099 (1948).

3 Chu, B. Laser Light Scattering 2nd edn (Academic Press, New York, 1991).

4 Berry, G. C. in Soft-Matter Characterization Vol. 1 (eds Borsali, R. \& Pecora, R.) 41-132 (Springer, Würzburg, 2008).

5 Chu, B. Laser light scattering. Annu. Rev. Phys. Chem. 21, 145-174 (1970).

6 Ezquerra, T. A., Garcia-Gutierrez, M. C., Nogales, A. \& Gomez, M. Applications of Synchrotron Light to Scattering and Diffraction in Materials and Life Sciences (Springer, Berlin and Heidelberg, 2009).

7 Squires, G. L. Introduction to The Theory of Thermal Neutron Scattering (Cambridge University Press, Cambridge, 1978).

8 Higgins, J. S. \& Benoit, H. C. Polymers and Neutron Scattering (Clarendon Press, Oxford, 1994).

9 Guinier, A. \& Fournet, G. Small-Angle Scattering of X-rays (John Wiley \& Sons, New York, 1955).

10 Borsali, R. in Light Scattering: Principles and Development (ed Brown, W.) (Oxford Univ. Press, Oxford, 1996).

11 Kirste, R. G., Kruse, W. A. \& Schelten, J. Die bestimmung des tragheitsradius von polymethylmethacrylat im glaszustand durch neutronenbeugung. Makromol. Chem. 162, 299-303 (1973).

12 Schelten, J., Wignall, G. D. \& Ballard, D. G. H Chain conformation in molten polyethylene by low angle neutron scattering. Polymer 15, 682-685 (1974).

13 Shibayama, M. in Soft Matter Characterization, Vol. 2 (eds Pecora, P. \& Borsali, R.) Ch. 14, 783-832 (Springer-Verlag, Würzburg, 2008).

14 Shibayama, M., Tanaka, T. \& Han, C. C. Small angle neutron scattering study on Poly(N-isopropyl acrylamide) gels near their volume-phase transition temperature. J. Chem. Phys. 97, 6829-6841 (1992).

15 Shibayama, M., Tanaka, T. \& Han, C. C. Small-angle neutron scattering study on weakly charged temperature sensitive polymer gels. J. Chem. Phys. 97, 6842-6854 (1992).

16 Shibayama, M. Spatial inhomogeneity and dynamic fluctuations of polymer gels. Macromol. Chem. Phys. 199, 1-30 (1998).

17 Shibayama, M., Isono, K., Okabe, S., Karino, T. \& Nagao, M. SANS study on pressureinduced phase separation of Poly(N-isoproptylacylamide) aqueous solutions and gels. Macromolecules 37, 2909-2918 (2004).

18 Nasimova, I. R., Karino, T., Okabe, S., Nagao, M. \& Shibayama, M. Small-angle neutron scattering investigation of pressure influence on the structure of weakly charged Poly(N-isopropyl acrylamide) solutions and gels. Macromolecules 37, 87218729 (2004).

19 Nasimova, I. R., Karino, T., Okabe, S., Nagao, M. \& Shibayama, M. Effect of ionization on the temperature and Pressure-induced phase transitions of poly( $\mathrm{N}$-isopropylacrylamide) gels and solutions. J. Chem. Phys. 121, 9708-9715 (2004).

20 Osaka, N. \& Shibayama, M. Pressure-induced phase transitions of hydrophobically solvated block copolymer solutions. Phys. Rev. Lett. 96, 048303 (2006).

21 Osaka, N., Miyazaki, S., Okabe, S., Endo, H., Sasai, A., Seno, K., Aoshima, S. \& Shibayama, M. Pressure-induced reentrant micellization of amphiphilic block copolymers in dilute aqueous solutions. J. Chem. Phys. 127, 094905 (2007). 
22 Osaka, N., Shibayama, M., Kikuchi, T. \& Yamamuro, O. Micro- and macrophase separations of hydrophobically solvated block copolymer aqueous solutions induced by pressure and temperature. Macromolecules 39, 5875-5884 (2006).

23 Tanaka, T. Collapse of gels and the critical endpoint. Phys. Rev. Lett. 40, 820-823 (1978).

24 Tanaka, T. Gels. Sci. Am. 244, 110-138 (1981).

25 Shibayama, M. \& Tanaka, T. Volume phase transition and related phenomena of polymer gels. Adv. Polym. Sci. 109, 1-62 (1993).

26 Hirokawa, Y. \& Tanaka, T. Volume phase transition in a nonionic gel. J. Chem. Phys. 81, 6379-6380 (1984)

27 Pelton, R. H. \& Chibante, P. Preparation of aqueous latices with $\mathrm{N}$-isopropylacrylamide. Colloids Surf. A Physicochem. Eng. Asp. 20, 247-256 (1986).

28 Schild, H. G. Poly(N-isopropylacrylamide): experiment, theory and application. Prog. Polym. Sci. 17, 163-249 (1992).

29 Hirotsu, S., Hirokawa, Y. \& Tanaka, T. Volume-phase transitions of ionized N-isopropylacrylamide gels. J. Chem. Phys. 87, 1392-1395 (1987).

$30 \mathrm{Li}, \mathrm{Y}$. \& Tanaka, T. Study of the universality class of the gel network system. J. Chem Phys. 90, 5161-5166 (1989).

31 Hirotsu, S. Elastic anomaly near the critical point of volume phase transition in polymer gels. Macromolecules 23, 903 (1990).

32 Hirotsu, S. Coexistence of phases and the nature of first-order phase transition in PolyN-isopropylacrylamide gels. Adv. Polym. Sci. 110, 1-26 (1993).

33 Bates, F. S. \& Fredrickson, G. H. Block copolymer thermodynamics: theory and experiment. Annu. Rev. Phys. Chem. 41, 525-557 (1990).

34 Shibayama, M., Ikkai, F., Inamoto, S., Nomura, S. \& Han, C. C. pH and salt concentration dependence of the microstructure of Poly( $\mathrm{N}$-isopropylacrylamideco-acrylic acid) gels. J. Chem. Phys. 105, 4358-4366 (1996).

35 Ikkai, F., Iritani, O., Shibayama, M. \& Han, C. C. Effect of degree of cross-linking on spatial inhomogeneity in charged gels. 3. Ionized effect. Macromolecules $\mathbf{3 1}$, 8526-8530 (1998).

36 Dusek, K. \& Prins, W. Structure and elasticity of non-crystalline polymer networks. Adv. Polym. Sci. 6, 1-102 (1969).

37 Candau, S., Bastide, J. \& Delsanti, M. Structural, elastic, and dynamic properties of swollen polymer networks. Adv. Polym. Sci. 44, 27 (1982).

38 Bastide, J. \& Leibler, L. Large-scale heterogeneities in randomly cross-linked networks. Macromolecules 21, 2647 (1988)

39 Panyukov, S. \& Rabin, Y. Statistical physics of polymer gels. Phys. Rep. 269, 1-132 (1996).

40 Panyukov, S. \& Rabin, Y. Polymer gels: frozen inhomogeneities and density fluctuations. Macromolecules 29, 7960 (1996).

41 Rabin, Y. \& Panyukov, S. Scattering profiles of charged gels: frozen inhomogeneities, thermal fluctuaitons, and microphase separation. Macromolecules 30, 301-312 (1997).

42 Panyukov, S. \& Rabin, Y. Volume transitions, phase separation, and anisotropic surface phases in charged gels. Macromolecules 29, 8530-8537 (1996).

43 Takata, S., Norisuye, T. \& Shibayama, M. Small-angle neutron scattering study on preparation temperature dependence of thermosensitive gels. Macromolecules 35, 4779-4784 (2002).

44 Shibayama, M., Takata, S. \& Norisuye, T. Static inhomogeneities and dynamic fluctuations of temperature sensitive polymer gels. Physica A 249, 245-252 (1998).

45 Onuki, A. Scattering from deformed swollen gels with heterogeneities. J. Phys. II France 2, 45-61 (1992).

46 Shibayama, M., Kawakubo, K., Ikkai, F. \& Imai, M. Small-angle neutron scattering study on charged gels in deformed state. Macromolecules $\mathbf{3 1}$, 2586-2592 (1998).

47 Hadziioannou, G., Wang, L. H., Stein, R. S. \& Porter, R. S Small-angle neutron scattering studies on amorphous polystyrene oriented by solid-state coextrusion. Macromolecules 15, 880-882 (1982).

48 Mendes, E. J., Lindner, P., Buzier, M., Boue, F. \& Bastide, J. Experimental evidence for inhomogeneous swelling and deformation in statistical gels. Phys. Rev. Lett. 66 , 1595 (1991)

49 Mendes, E., Oeser, R., Hayes, C., Boue, F. \& Bastide, J. Small-angle neutron scattering study of swollen elongated gels: butterfly patterns. Macromolecules 29, 5574 (1996).

50 Mendes, E., Schosseler, F., Isel, F., Boue, F., Bastide, J. \& Candau, S. J. A sans study of uniaxially elongated polyelectrolyte gels. Europhys. Lett. 32, 273 (1995).

51 Geissler, E., Duplessix, R. \& Hecht, A. M Radius of gyration in anisotropically swollen gels. Macromolecules 16, 712-713 (1983).

52 Shibayama, M., Kawakubo, K. \& Norisuye, T. Comparison of the experimental and theoretical structure factors of temperature sensitive polymer gels. Macromolecules 31, 1608-1614 (1998)

53 Shibayama, M., Ikkai, F., Shiwa, Y. \& Rabin, Y. Effect of degree of cross-linking on spatial inhomogeneity in charged gels. I. theoretical prediction and light scattering study. J. Chem. Phys. 107, 5227-5235 (1997)

54 Ikkai, F. \& Shibayama, M. Anomalous cross-link density dependence of scattering from charged gels. Phys. Rev. E 56, R51-R54 (1997).

55 Ikkai, F. \& Shibayama, M. Static inhomogeneities in thermoreversible gels. Phys. Rev. Lett. 82, 4946-4949 (1999).

56 Osaka, N., Takata, S., Suzuki, T., Endo, H. \& Shibayama, M. Comparison of heat- and pressure-induced gelation of $\beta$-lactoglobulin aqueous solutions studied by small-angle neutron and dynamic light scattering. Polymer 49, 2957-2963 (2008).

57 Matsumoto, M., Murakoshi, K., Wada, Y. \& Yanagida, S. High pressure effects on fluorescence-quenching of coumarin 343 adsorbed on $\mathrm{TiO}_{2}$ nanocystallites in methanol and in N,N-dimehtylformamide. Chem. Lett. 29, 938-939 (2000).
58 Otake, K., Karaki, R., Ebina, T., Yokoyama, C. \& Takahashi, S. Pressure effects on the aggregation of poly( $\mathrm{N}$-isopropylacrylamide) and poly( $\mathrm{N}$-isopropylacrylamide-co-acrylic acid) in aqueous solutions. Macromolecules 26, 2194-2197 (1993).

59 Kato, E. Volume-phase transition of $\mathrm{N}$-isopropylacrylamide gels induced by hydrostatic pressure. J. Chem. Phys. 106, 3792-3797 (1997).

60 Rebelo, L. P. N., Visak, Z. P., de Sousa, H. C., Szydlowski, J., Gomes de Azevedo, R. Ramos, A. M., Najdanovic-Visak, V., Nunes da Ponte, M. \& Klein, J. Double critical phenomena in (water+polyacrylamides) solutions. Macromolecules 35, 1887 (2002).

61 Smeller, L. Pressure-temperature phase diagram of biomolecules. Biochim. Biophys. Acta 1595, 11-29 (2002).

62 Kato, E. Thermodynamic study of a pressure-temperature phase diagram for poly $(\mathrm{N}$ isopropylacrylamide) gels. J. Appl. Polym. Sci. 97, 405-412 (2005).

63 Okumura, Y. \& Ito, K. The polyrotaxane gel: a topological gel by figure-of-eight crosslinks. Adv. Mater. 13, 485-487 (2001).

64 Ball, R. C., Edwards, S. F., Doi, M. \& Warner, M. Elasticity of entangled networks. Polymer 22, 1010-1018 (1981).

65 Ito, K. Novel cross-linking concept of polymer network: synthesis, structure, and properties of slide-ring gels with freely movable junctions. Polym. J. 39, 489-499 (2007)

66 Haraguchi, K. \& Takehisa, T. Nanocomposite hydrogels: a unique organic-inorganic network structure with extraordinary mechanical, optical, and swelling/de-swelling properties. Adv. Mater. 14, 1120-1124 (2002).

67 Gong, J. P., Katsuyama, Y., Kurokawa, T. \& Osada, Y. Double-network hydrogels with extremely high mechanical strength. Adv. Mater. 15, 1155-1158 (2003)

68 Sakai, T., Matsunaga, T., Yamamoto, Y., Ito, C., Yoshida, R., Suzuki, S., Sasaki, N., Shibayama, M. \& Chung, U. Design and fabrication of a high-strength hydrogel with ideally homogeneous network structure from tetrahedron-like macromonomers. Macromolecules 41, 5379-5384 (2008).

69 Schmidt, H. in Polymer Based Molecular Composites (eds D. W. Schaefer \& J. E. Mark) Mater. Res. Soc. (1990).

70 Novak, B. M Hybrid nanocomposite materials-between inorganic glasses and organic polymers. Adv. Mater. 5, 422-433 (1993).

71 Haraguchi, K., Takehisa, T. \& Fan, S. Effects of clay content on the properties of nanocomposite hydrogels composed of poly( $\mathrm{N}$-isopropylacrylamide) and clay. Macromolecules 35, 10162-10171 (2002).

72 Haraguchi, K., Farnworth, R., Ohbayashi, A. \& Takehisa, T. Compositinal effects on mechanical properties of nanocomposite hydrogels composed of poly $(\mathrm{N}, \mathrm{N}$-dimthylacrylamide) and clay. Macromolecules 36, 5732-5741 (2003).

73 Haraguchi, K., Li, H.- J., Matsuda, K., Takehisa, T. \& Elliot, E. Mechanism of forming organic/inorganic network structures during in-situ free-radical polymerization in PNIPA-clay nanocomposite hydrogels. Macromolecules 38, 3482-3490 (2005).

74 Shibayama, M., Suda, J., Karino, T., Okabe, S., Takehisa, T. \& Haraguchi, K. Structure and dynamics of poly( $\mathrm{N}$-isopropyl acrylamide)-clay nanocomposite gels. Macromolecules 37, 9606-9612 (2004).

75 Miyazaki, S., Karino, T., Endo, H., Haraguchi, K. \& Shibayama, M. Clay concentration dependence of microstructure in deformed poly( $\mathrm{N}$-isopropylacrylamide)-clay nanocomposite gels. Macromolecules 39, 8112-8120 (2006).

76 Miyazaki, S., Endo, H., Karino, T., Haraguchi, K. \& Shibayama, M. Gelation mechanism of poly(N-isopropylacrylamide)-clay nanocomposite gels. Macromolecules 40, 4287-4295 (2007)

77 Endo, H., Miyazaki, S., Haraguchi, K. \& Shibayama, M. Structure of nanocomposite hydrogel investigated by means of contrast variation small angle neutron scattering. Macromolecules 41, 5406-5411 (2008).

78 Shibayama, M., Karino, T., Miyazaki, S., Takehisa, T. \& Haraguchi, K. Small-angle neutron scattering study on uniaxially stretched poly( $\mathrm{N}$-isopropylacrylamide)-clay nanocomposite gels. Macromolecules 38, 10772-10781 (2005).

79 Nishida, T., Endo, H., Osaka, N., Li, H. J., Haraguchi, K. \& Shibayama, M. Deformation mechanism of nanocomposite gels studied by contrast variation smallangle neutron scattering. Phys. Rev. E 80, 030801 (2009).

80 Karino, T., Okumura, Y., Ito, K. \& Shibayama, M. SANS studies on spatial inhomogeneities of slide-ring gels. Macromolecules 37, 6117-6182 (2004).

81 Haraguchi, K. \& Li, H.- J. Mechanical properties and structure of polymer-clay nanocomposite gels with high clay content. Macromolecules 39, 1898-1905 (2006).

82 Shibayama, M. Miyazaki, S., Endo, H., Karino, T. \& Haraguchi, K. Deformation studies on polymer-clay nanocomposite gels. Macromol. Symp. 256, 131-136 (2007)

83 Endo, H., Schwahn, D. \& Cölfen, J. On the role of block copolymer additives for calcium carbonate crystallization: small angle neutron scattering investigation by applying contrast variation. J. Chem. Phys. 120, 9410-9423 (2004).

84 Osaka, N., Endo, H., Nishida, T., Suzuki, T., Li, H. J., Haraguchi, K. \& Shibayama, M. Microphase separation in nanocomposite gels. Phys. Rev. E Rapid Commun. 79, 060801 (2009).

85 Kurakazu, M., Katashima, T., Chijiishi, M., Nishi, N., Akagi, Y., Matsunaga, T., Shibayama, M., Chung, U. \& Sakai, T. Evaluation of gelation kinetics of Tetra-PEG gel. Macromolecules 43, 3935-3940 (2010).

86 Matsunaga, T., Sakai, T., Akagi, Y., Chung, U. \& Shibayama, M. Structure characterization of Tetra-PEG Gel by small-angle neutron scattering. Macromolecules 42, 1344-1351 (2009).

87 Akagi, Y., Matsunaga, T., Shibayama, M., Chung, U. \& Sakai, T. Evaluation of topological defects in Tetra-PEG gels. Macromolecules 43, 488-493 (2010).

88 Akagi, Y. et al. Effect of module size on the network structure of Tetra-PEG gel (in preparation). 
89 Bastide, J. \& Candau, S. J. in The Physical Properties of Polymer Gels Ch. 9 (ed Cohen Addad, J. P.) 143 (John Wiley, New York, 1996).

90 Mendes, E., Hakiki, A., Herz, J., Boue, F. \& Bastide, J. Structure of trifunctional endlink polymer gels studied by SANS. Macromolecules 37, 2643-2649 (2004).

91 Sukumaran, S. K., Beaucage, G., Mark, J. E. \& Viers, B. Neutron scattering from equilibrium-swollen networks. Eur. Phys. J. E 18, 29-36 (2005).

92 Sakai, T., Akagi, Y., Matsunaga, T., Kurakazu, M., Chung, U. \& Shibayama, M. Highly elastic and deformable hydrogel formed from tetra-arm polymers. Macromol. Rapid Commun. 31, 1954-1959 (2010).

93 Matsunaga, T., Sakai, T., Akagi, Y., Chung, U. \& Shibayama, M. SANS and SLS studies on tetra-arm PEG gels in as-prepared and swollen states. Macromolecules 42, 6245-6252 (2009)

94 Hammouda, B., Ho, D. \& Kline, S. SANS from poly(ethylene oxide)/water systems. Macrmolecules 35, 8578-8585 (2002).

95 Hammouda, B., Ho, D. L. \& Kline, S. Insight into clustering in poly(ethylene oxide) solutions. Macrmolecules 37, 6932-6937 (2004)
96 Haraguchi, K., Xu, Y. \& Li, G. Molecular characteristics of poly(N-isopropylacrylamide) separated from nanocomposite gels by removal of clay from the polymer/clay network. Macromol. Rapid Commun. 31, 718-723 (2010).

$97 \mathrm{Xu}, \mathrm{Y} ., \mathrm{Li}, \mathrm{G}$. \& Haraguchi, K. Gel formation and molecular characteristics of poly(N-isopropylacrylamide) prepared by free-radical redox polymerization in aqueous solution. Macromol. Chem. Phys. 211, 977-987 (2010).

98 Shibayama, M., Matsunaga, T. \& Nagao, M. Evaluation of incoherent scattering intensity by transmission and sample thickness. J. Appl. Cryst. 42, 621-628 (2009).

99 Shibayama, M., Takahashi, H. \& Nomura, S. Small-angle neutron scattering study on end-linked poly(tetrahydrofuran) networks. Macromolecules 28, 6860-6864 (1995).

100 Villar, M. A. \& Valles, E. M Influence of pendant chains on mechanical properties of model poly(dimethylsiloxane) networks .2. Viscoelastic properties. Macromolecules 29, 4081 (1996).

101 Granick, S. \& Rubinstein, M. A multitude of macromolecules. Nat. Mater. 3, 586-587 (2004)

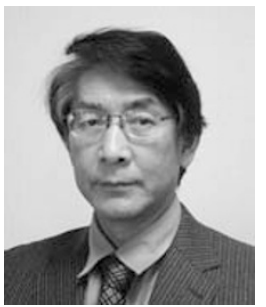

Mitsuhiro Shibayama was born in 1954. He received his B. Eng., M. Eng. and Dr Eng. in Polymer Chemistry from Kyoto University, in 1977, 1979 and 1983, respectively. After serving as a JSPS fellow and as a research associate at the University of Massachusetts at Amherst, he joined Kyoto Institute of Technology in 1984. He was promoted to the position of associate professor in 1988 and as full professor in 1997. He spent a sabbatical year in MIT in 1991. In 2000, he moved to Neutron Scattering Laboratory, Institute for Solid State Physics, the University of Tokyo. His research interest covers structure investigation and dynamics of soft matter with various scattering techniques. He has received the Sakurada Takeshi Memorial Award (Japan Fiber Society, 1991), the Wiley Science Award (Society of Polymer Science, Japan, 2000) and the Chemical Society of Japan Award for Creative Work, 2004, as well as the Award of the Society of Polymer Science, Japan, 2010. 